\title{
THE STRUCTURE OF MFD SHOCK WAVES FOR RECTILINEAR MOTION IN SOME MODELS OF PLASMA
}

\author{
MAHMOUD HESAARAKI
}

\begin{abstract}
The mathematical question of the existence of structure for "fast", "slow" and "intermediate" MFD shock waves in the case of rectilinear motion in some model of plasma is stated in terms of a six-dimensional system of ordinary differential equations, which depends on five viscosity parameters. In this article we shall show that this system is gradient-like. Then by using the Conley theory we prove that the fast and the slow shocks always possess structure. Moreover, the intermediate shocks do not admit structure. Some limiting cases for singular viscosities are investigated. In particular, we show how the general results in the classical one fluid MHD theory are obtained when "the plasma viscosities" $\beta$ and $\chi$ tend to zero.
\end{abstract}

\section{INTRODUCTION}

In this article we shall continue our work, begun in [12] for general cases on the structure of MFD shock waves, of arbitrary strength for rectilinear motion of a fluid which is, as a whole, electrically neutral. Two types of particles are present: ions (mass $m_{1}$, charge $e$ ) and electrons (mass $m_{2}$, charge $-e$ ). It is assumed that stresses are defined by a scalar pressure and that thermodynamical properties are those of an ordinary simple fluid.

As discussed in [9] and [10], the structure problem reduces to finding heteroclinic orbits for a six-dimensional system of ordinary differential equations as given in equations (1.1). For this system the independent variable $x$ is a coordinate variable. The constant $B_{1}$ is the longitudinal component of the magnetic induction, while the variables $B_{2}$ and $B_{3}$ are the components of this field on the $y$ - and $z$-axes. The characters $m=m_{1}+m_{2}, P>0, \mu>0, E_{2}, E_{3}$ and $C$ are all constants. The constants $E_{2}$ and $E_{3}$ are the components of electrical fields on the $y$ - and $z$-axes. The variable $u$ is the velocity which is related to the specific volume of the fluid, $V$ by the equality $u=m V$, while $T, p$ and $e$ correspond to temperature, pressure and internal energy, respectively; $\xi_{2}$ and $\xi_{3}$ are auxiliary variables which are defined by the first two equations

Received by the editors June 7, 1993 and, in revised form, September 6, 1994.

1991 Mathematics Subject Classification. Primary 76L05, 76X05, 58F40; Secondary 76W05, 58F25, 34A10.

Key words and phrases. Plasma, shock wave structure, heteroclinic orbit, Conley theory.

This work was done while the author was on Sabbatical leave visiting the International Centre for Theoretical Physics, Trieste, Italy. 
of the system (1.1). The symbols $\eta, k$ and $\sigma^{-1}$ are the viscosity, the heat conduction and the Joule's dissipation, respectively; $\beta$ and $\chi$ are two coefficients given by $\beta=m_{1} m_{2}\left(\mu e^{2} m\right)^{-1}$ and $\chi=\left(m_{1}-m_{2}\right)(\mu e m)^{-1}$. We call $\eta$, $k, \sigma^{-1}, \beta$ and $\chi$ the viscosity parameters. The viscosities $\eta, k, \sigma^{-1}$ and $\beta$ are nonnegative, but $\chi \in R$. For the derivation of these equations, we refer the reader to Chapter 4 in [1].

$$
\begin{aligned}
u \frac{d B_{2}}{d x}= & \xi_{2}, \\
u \frac{d B_{3}}{d x}= & \xi_{3} \\
\beta u \frac{d \xi_{2}}{d x}= & E_{3}+\left(u-\frac{B_{1}^{2}}{m \mu}\right) B_{2}-\chi B_{1} \xi_{3}-(\sigma \mu)^{-1} \frac{\xi_{2}}{u} \\
\beta u \frac{d \xi_{3}}{d x}= & -E_{2}+\left(u-\frac{B_{1}^{2}}{m \mu}\right) B_{3}+\chi B_{1} \xi_{2}-(\sigma \mu)^{-1} \frac{\xi_{3}}{u}, \\
\eta \frac{d u}{d x}= & p+m u+\frac{1}{2 \mu}\left(B_{2}^{2}+B_{3}^{2}\right)-P, \\
k \frac{d T}{d x}= & m e-\frac{1}{2} m u^{2}+\left(\frac{B_{1}^{2}}{2 m \mu^{2}}-\frac{u}{2 \mu}\right)\left(B_{2}^{2}+B_{3}^{2}\right)+P u \\
& +\frac{\beta}{2 \mu}\left(\xi_{2}^{2}+\xi_{3}^{2}\right)-\frac{1}{\mu}\left(E_{3} B_{2}-E_{2} B_{3}\right)-m C .
\end{aligned}
$$

The above system of equations has been studied before by Germain in [9] and [10] and by Peyret in [17] and [18] for "fast" and "slow" shocks when at least $\eta=k=0$. However, in these papers no discussion has been given on the stability of the flow, even for these special cases.

This system also has been studied by the author in [12] and [15] for "fast", "slow", "intermediate", "switch-on" and "switch-off" shocks whenever $B_{1}=0$ or $\chi=0$. For these cases the stability of the flow have been shown in both papers [12] and [15].

For all the above special cases the system of six equations reduces to a system of four equations.

This leaves open the question of the structures for the fast, the slow and the intermediate shocks where all of the viscosity parameters are positive and $B_{1} \neq 0$.

In order to handle this and related problems, we shall show that the system (1.1) is gradient-like with respect to a real-valued function $P$ (except for a very special case). In view of the gradient-like property each isolated rest point is an "isolated invariant set" the nondegenerate one has the Conley index different from $\overline{0}$ (namely, the homotopy class of the one-point pointed space). Another crucial fact is that the values of $P$ at the rest points are the same as the entropy of the system at these points.

With these facts established, the existence of structure reduces to showing that for all viscosity values, the relevant rest points are contained in a large isolated invariant set with the Conley index $\overline{0}$. This topological aspect is discussed and appropriate references to the notions involved are given in $\S 3$. 
In $\S 2$ we will study the nature of the rest points of the system (1.1). In $\S 3$ we shall build an "isolating neighbourhood" containing all complete bounded orbits. In $\S 4$ we will prove the existence of structure for the fast and the slow shocks. In $\S 5$ we see that the intermediate shocks need not always possess structure. Finally, some limiting cases, i.e., where some of the viscosity parameters are zero, will be considered in $\S 6$.

We will make use of Conley and Smoller's results in [4] and [5] extensively from the next section in our work. Because of this, we present the following statements to examine the difference and the relationship between Conley and Smaller's work in [4] and [5] and our work in this paper.

1. In both works the fluid is as a whole electrically neutral. In Conley and Smoller's work each particle in the fluid is electrically neutral too (because of this, they have the word "hydro" in the titles of their papers). But in our work each particle in the fluid is ionized as mentioned before (because of this the word "plasma" appeared in the title of the paper). This fact causes the appearance of the dissipation coefficients $\beta$ and $\chi$ and the related terms in our equations (1.1).

2. In both works the fluid motion is one dimensional (the $x$-axis direction in our work). In our work each particle in the fluid moves just in the direction of the $x$-axis too. But in the Conley and Smoller's work each particle in the fluid can move in the three-dimensional space. Because of this fact, they have the dissipation coefficient $\mu$ and the related terms in their equations (2.2) in [4].

Now if we let $\beta=\chi=0$ in our equations (1.1), we obtain the system of four equations (3.5) in $\S 3$, while if we let $\mu=0$ in their equations (2.2) in [4] we obtain the equations (3.5) in $\S 3$ too. Hence the rest points for both of the system of equations (1.1) and the system of equations (2.2) in [4] are the same. These relationships between the system (1.1) and the system (2.2) in [4] enable us to take advantage of some results from [4] and [5] in our work.

\section{THE REST POINTS AND THE PROBLEMS}

Consider the system (1.1). As Kulikovskiy and Lyubimov mentioned in [16], this system of differential equations is invariant under rotation of the reference coordinate system about the $x$-axis. Thus we may assume that $E_{2}=0$ and $E_{3} \geq 0$. Since $m$ is the mass quantity and $\mu$ is an electrical quantity, without loss of generality we may assume that $\mu=m=1$. Then $u=m V$ implies $u=V$. Also in order to avoid confusion in notation, and our work makes extensive use of Conley and Smoller's results in [4] and [5]; and of our previous works in [12], [13] and [15], we replace $x, \beta^{1 / 2}, \beta^{1 / 2} \xi_{i}, E_{3}, P$ and $B_{1}$ by $t, \alpha, y_{i}, \varepsilon, J$ and $\delta, i=2,3$, respectively. Using these the system (1.1) becomes

$$
A \dot{u}=G(u) \quad\left(\cdot=\frac{d}{d t}\right)
$$

where $A=\operatorname{diag}(\alpha V, \alpha V, \alpha V, \alpha V, \eta, k)^{\mathrm{T}}, u=\left(B_{2}, B_{3}, y_{2}, y_{3}, V, T\right)^{\mathrm{T}}$ and $G(u)=\left(G_{1}(u), G_{2}(u), G_{3}(u), G_{4}(u), G_{5}(u), G_{6}(u)\right)^{\mathrm{T}}$ with 


$$
\begin{aligned}
G_{1}(u)= & y_{2}, \\
G_{2}(u)= & y_{3}, \\
G_{3}(u)= & \varepsilon+\left(V-\delta^{2}\right) B_{2}-\chi \alpha^{-1} \delta y_{3}-\frac{\sigma^{-1} y_{2}}{\alpha V}, \\
G_{4}(u)= & \left(V-\delta^{2}\right) B_{3}+\chi \alpha^{-1} \delta y_{2}-\frac{\sigma^{-1} y_{3}}{\alpha V}, \\
G_{5}(u)= & p(V, T)+V+\frac{1}{2}\left(B_{2}^{2}+B_{3}^{2}\right)-J, \\
G_{6}(u)= & e(V, T)-\frac{1}{2} V^{2}-\frac{1}{2}\left(V-\delta^{2}\right)\left(B_{2}^{2}+B_{3}^{2}\right) \\
& +J V+\frac{1}{2}\left(y_{2}^{2}+y_{3}^{2}\right)-\varepsilon B_{2}-C .
\end{aligned}
$$

From now on we assume that $\delta \neq 0$ and $\varepsilon>0$. For the case $\delta=0$, the reader is referred to [15]. The case $\varepsilon=0$ will be considered in a future publication.

Let $S(V, T)$ be the entropy of the system. Following [4], [5], [12], [13], [14] and [15] we assume that the following hypotheses hold:

$H_{1}$ : The functions $p, e$ and $S$ are positive when $V, T>0$.

$H_{2}:$ For fixed $T>0, p(V, T) \rightarrow+\infty$ as $V \rightarrow 0$.

$H_{3}$ : Given positive constants $K$ and $V_{0}$, there exists $T_{0}>0$ such that if $0<V \leq V_{0}$ and $T \geq T_{0}$, then $e(V, T)>K$.

$H_{4}$ : On any interval $0<V \leq V_{0}, S(V, T) \rightarrow 0$ uniformly in $V$ as $T \rightarrow 0$. As an alternative to $H_{4}$, we could assume:

$H_{4}^{\prime}: S_{V}\left(=p_{T}\right)$ and $S_{T}$ are positive whenever $V, T>0$, and for any fixed $V, S(V, T)$ converges to a limit independent of $V$ as $T \rightarrow 0$.

$H_{5}: P_{V}<0, p_{V V}>0$ and $p_{S}>0$, wherein $p$ is considered as a function of $V$ and $S$.

We shall not use all of these hypotheses directly, but we will take advantage of some results based on them.

In order to introduce the problem, it is necessary to make some observations concerning the rest points.

At a rest point $y_{2}=y_{3}=B_{3}=0$ and $B_{2}=-\varepsilon /\left(V-\delta^{2}\right)$. Substituting these values of $y_{i}$ and $B_{i}, i=2,3$, into $G_{5}(u)=G_{6}(u)=0$, we arrive at the following criterion for a rest point:

$$
\begin{aligned}
& F_{1}(V, T)=\frac{1}{2} \varepsilon^{2}\left(V-\delta^{2}\right)^{-2}+V-J+p(V, T)=0, \\
& F_{2}(V, T)=\frac{1}{2} \varepsilon^{2}\left(V-\delta^{2}\right)^{-1}-\frac{1}{2} V^{2}+J V-C+e(V, T)=0 .
\end{aligned}
$$

With the hypothesis $p_{T}>0$, we have $\partial F_{1} / \partial T>0$. Also $S_{T}>0$ together with the second law of thermodynamics (i.e., $d e=-p d V+T d S$ ) implies $\partial F_{2} / \partial T=e_{T}=T S_{T}>0$. Thus $F_{1}(V, T)=0$ and $F_{2}(V, T)=0$ determine the graphs of functions $T_{1}(V)$ and $T_{2}(V)$, respectively in the $V, T$ plane. These functions are not defined at $V=\delta^{2}$, which divides each of the graphs into two branches (see Figure 2.1).

From the appendix in [4] we have the following theorem. (See also [12].) 


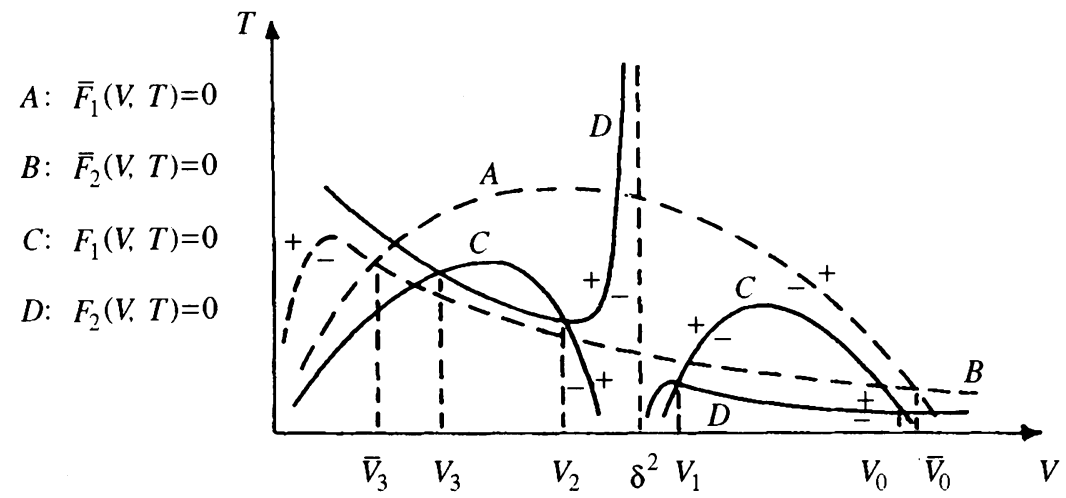

Figure 2.1. The + and - signs show the sign of the related function to the curve in the regions

Theorem 2.1. For fixed values of $J, \delta$ and $\varepsilon$, there are numbers $C_{0} \geq C_{1}$ such that for $C>C_{0}$ the system of algebraic equations (2.3) admits no solution at all. For $C<C_{1}$ it admits precisely four solutions. Two of these solutions are located in the region $V>\delta^{2}$ and the other two are located in the region $0<V<\delta^{2}$. For $C=C_{0}, C_{1}<C<C_{0}$ and $C=C_{1}$, it admits one, two and three solutions, respectively. At most two of these solutions are located in $V>\delta^{2}$ (or in $0<V<\delta^{2}$ ).

For the proof of the above theorem the reader is referred to [4] with the hint that by changing $C$ to $E$ in our equations (2.3) we obtain the equations (2.4) in [4]. These equations are the same as (1.2.1) in [13].

From now on we assume that the system of algebraic equation (2.3) admits four solutions. We call them $\left(V_{i}, T_{i}\right), 0 \leq i \leq 3$, where $V_{0}>V_{1}>\delta^{2}>V_{2}>$ $V_{3}$. This means that the system $(2.1)$ has four rest points

$$
u_{i}=\left(B_{2 i}, B_{3 i}, y_{2 i}, y_{3 i}, V_{i}, T_{i}\right), \quad 0 \leq i \leq 3,
$$

with $B_{2 i}=-\varepsilon\left(V_{i}-\delta^{2}\right)^{-1}$ and $B_{3 i}=y_{2 i}=y_{3 i}=0$.

In order to show that all of the above rest points are nondegenerate and $S\left(V_{i}, T_{i}\right)<S\left(V_{i+1}, T_{i+1}\right), 0 \leq i \leq 2$, we recall the following results from $\S 1.2$ in [13].

Theorem 2.2. (i) $\frac{d T_{1}}{d V}=0$, precisely once in each of its branches, which are both maximum. (ii) The curves $\left\{(V, T): F_{2}(V, T)=0, V_{i+1}<V<V_{i}\right\}, i=0,2$, lie in the region $\left\{(V, T): F_{1}(V, T)<0\right\}$. (iii) $T_{2}(V)$ is decreasing in the intervals $\left(V_{i+1}, V_{i}\right), i=0$ and 2 .

For the case $\varepsilon=0$ equations (2.3) reduce to the following

$$
\begin{aligned}
& \bar{F}_{1}(V, T)=V-J+p(V, T)=0, \\
& \bar{F}_{2}(V, T)=e(V, T)-\frac{1}{2} V^{2}+J V-C=0 .
\end{aligned}
$$

Similar to $(2.3), \bar{F}_{1}(V, T)=0$ and $\bar{F}_{2}(V, T)=0$ determine the graphs of functions $\bar{T}_{1}(V)$ and $\bar{T}_{2}(V)$, respectively. From $\S 3.3$ in [13] we have the following results. 
Theorem 2.3. (i) $d \bar{T}_{1} / d V=0$, precisely once. This is a maximum. (ii) If the system of equations (2.3) admits four solutions, then (2.5) admits two solutions say $\left(\bar{V}_{i}, \bar{T}_{i}\right), i=0,3, \bar{V}_{3}<\bar{V}_{0}$. Moreover $\bar{V}_{3}<V_{3}<V_{2}<\delta^{2}<V_{1}<V_{0}<$ $\bar{V}_{0}$. (iii) $\bar{T}_{2}(V)$ is decreasing in the interval $\left[\bar{V}_{3}, \bar{V}_{0}\right]$. (iv) For $V \in\left[V_{3}, V_{2}\right]$ we have $\bar{T}_{2}(V)<T_{2}(V)$, but $V \in\left[V_{1}, V_{0}\right]$ implies $T_{2}(V)<\bar{T}_{2}(V)$.

For the proofs of these theorems the reader is referred to $\S \S 1.2$ and 3.3 in [13]. The graphs of $F_{i}(V, T)=0$ and $\bar{F}_{i}(V, T)=0, i=1,2$, are depicted in Figure 3.3.3 in [13]. We reproduce them in Figure 2.1.

We can now prove the following lemma.

Lemma 2.1. Let $S(u)=S(V, T)$ be the entropy of the system as before. (i) $S(u)$ is strictly decreasing along the curve $T_{2}(V)$ in the intervals $\left[V_{i+1}, V_{i}\right], i=0,2$, and along the curve $\bar{T}_{2}(V)$ in the interval $\left[\bar{V}_{3}, \bar{V}_{0}\right]$. (ii) $S\left(u_{i}\right)<S\left(u_{i+1}\right)$ for $i=0,1,2$.

Proof. (i) If we differentiate $F_{2}(V, T)=0$ with respect to $V$, we get

$$
-\frac{1}{2} \varepsilon^{2}\left(V-\delta^{2}\right)^{-2}-V+J+e_{V}+e_{T} \frac{d T_{2}}{d V}=0
$$

But by the second law of thermodynamics $e_{T}=T S_{T}$ and $e_{V}=T S_{V}-p$, so

$$
-\frac{1}{2} \varepsilon^{2}\left(V-\delta^{2}\right)^{-2}-V+J-p+T S_{V}+T S_{T} \frac{d T_{2}}{d V}=0 .
$$

On the other hand $-\frac{1}{2} \varepsilon^{2}\left(V-\delta^{2}\right)^{-2}-V+J-p=-F_{1}(V, T)$. Thus

$$
T\left(S_{V}+S_{T} \frac{d T_{2}}{d V}\right)=F_{1}\left(V, T_{2}(V)\right) .
$$

Then, by considering Theorem 2.2, it follows that for $u \in\left(V_{3}, V_{2}\right) \cup\left(V_{1}, V_{0}\right)$ we have

$$
S_{V}+S_{T} \frac{d T_{2}}{d V}<0
$$

Thus $\frac{d S}{d V}<0$ along the curve $T_{2}(V)$ in the intervals $\left(V_{i+1}, V_{i}\right), i=0,2$. Hence $S\left(V, T_{2}(V)\right)$ is strictly decreasing in these intervals. Therefore $S\left(V_{i+1}, T_{2}\left(V_{i+1}\right)\right)>S\left(V_{i}, T_{2}\left(V_{i}\right)\right)$ or $S\left(u_{i+1}\right)>S\left(u_{i}\right), i=0,2$. By using a similar argument, we can show that $S\left(V, \bar{T}_{2}(V)\right)$ is strictly decreasing in the interval $\left[\bar{V}_{3}, \bar{V}_{0}\right]$.

(ii) According to Theorem 2.3, $\bar{T}_{2}\left(V_{2}\right)<T_{2}\left(V_{2}\right)$ and $T_{2}\left(V_{1}\right)<\bar{T}_{2}\left(V_{1}\right)$. Since $S_{T}>0, S\left(V_{2}, T_{2}\left(V_{2}\right)\right)>S\left(V_{2}, \bar{T}_{2}\left(V_{2}\right)\right)$ and $S\left(V_{1}, \bar{T}_{2}\left(V_{1}\right)\right)>S\left(V_{1}, T_{2}\left(V_{1}\right)\right)$. But by the above $S\left(V, \bar{T}_{2}(V)\right)$ is strictly decreasing in $\left[\bar{V}_{3}, \bar{V}_{0}\right]$ and $\bar{V}_{3}<$ $V_{2}<V_{1}<\bar{V}_{0}$. Thus $S\left(V_{2}, \bar{T}_{2}\left(V_{2}\right)\right)>S\left(V_{1}, \bar{T}_{2}\left(V_{1}\right)\right)$. Hence $S\left(V_{2}, T_{2}\left(V_{2}\right)\right)>$ $S\left(V_{1}, T_{2}\left(V_{1}\right)\right)$ or $S\left(u_{2}\right)>S\left(u_{1}\right)$. Therefore $S\left(u_{i+1}\right)>S\left(u_{i}\right)$ for $i=0,1$ and 2 .

We will use the following lemma to show that all of the rest points $u_{i}$, $0 \leq i \leq 3$, are nondegenerate.

Lemma 2.2. At the rest point $u_{0}$ and $u_{2}, S_{T}\left[1+p_{V}-\varepsilon^{2}\left(V-\delta^{2}\right)^{-3}\right]-S_{V}^{2}$ is positive, while it is negative at the rest points $u_{1}$ and $u_{3}$.

Proof. Differentiating $F_{1}(V, T)=0$ with respect to $V$ yields

$$
-\varepsilon^{2}\left(V-\delta^{2}\right)^{-3}+1+p_{V}+p_{T} \frac{d T_{1}}{d V}=0
$$


or

$$
\frac{d T_{1}}{d V}=\frac{1}{p_{T}}\left[\varepsilon^{2}\left(V-\delta^{2}\right)^{-3}-1-p_{V}\right]
$$

On the other hand, $F_{1}\left(V, T_{2}(V)\right)=0$ at the rest points. This and (2.6) imply $\frac{d T_{2}}{d V}=\frac{1}{S_{T}}\left(-S_{V}\right)$, at these points. But from the nature of $T_{1}(V)$ and $T_{2}(V)$ (see Theorem 2.2 or Figure 2.1) we must have $\frac{d T_{2}}{d V}>\frac{d T_{1}}{d V}$ at $u_{0}$ and $u_{2}$ (< at $u_{1}$ and $u_{3}$ ). Then by using $p_{T}=S_{V}$ we get

$$
S_{T}\left[1+p_{V}-\varepsilon^{2}\left(V-\delta^{2}\right)^{-3}\right]-S_{V}^{2}>0 \text {, }
$$

at the rest points $u_{0}$ and $u_{2} \quad\left(<\right.$ at $u_{1}$ and $\left.u_{3}\right)$.

The linearized system of $(2.1)$ at the rest point $u_{i}$ can be written as $\dot{u}=$ $M_{i}\left(u-u_{i}\right)$ where

$$
M=\left[\begin{array}{cccccc}
0 & 0 & \frac{1}{\alpha V} & 0 & 0 & 0 \\
0 & 0 & 0 & \frac{1}{\alpha V} & 0 & 0 \\
\frac{V-\delta^{2}}{\alpha V} & 0 & -\frac{\sigma^{-1}}{\alpha^{2} V^{2}} & -\frac{\chi \delta}{\alpha^{2} V} & -\frac{\varepsilon}{\alpha V\left(V-\delta^{2}\right)} & 0 \\
0 & \frac{V-\delta^{2}}{\alpha V} & \frac{\chi \delta}{\alpha^{2} V} & -\frac{\sigma^{-1}}{\alpha^{2} V^{2}} & 0 & 0 \\
-\frac{\varepsilon}{\eta\left(V-\delta^{2}\right)} & 0 & 0 & 0 & \frac{1+p_{V}}{\eta} & \frac{S_{V}}{\eta} \\
0 & 0 & 0 & 0 & \frac{T S_{V}}{k} & \frac{T S_{T}}{k}
\end{array}\right]
$$

in which the subscript $i$ has been dropped and the identities $p_{T}=S_{V}, e_{V}=$ $T S_{V}-p$ and $e_{T}=T S_{T}$ have been used. Thus

$$
\operatorname{det} M=\frac{T\left(V-\delta^{2}\right)^{2}}{\alpha^{4} V^{4} k \eta}\left\{S_{T}\left[1+p_{V}-\frac{\varepsilon^{2}}{\left(V-\delta^{2}\right)^{3}}\right]-S_{V}^{2}\right\} \text {. }
$$

Then Lemma 2.2 implies that the product of the eigenvalues at $u_{0}$ and $u_{2}$ is positive and at $u_{1}$ and $u_{3}$ is negative. Thus we have proved the following theorem.

Theorem 2.4. Suppose in the system (2.1), $\delta \varepsilon \neq 0$ and this system admits four rest points. Then all of them are nondegenerate.

Now consider the system of $n$ conservation laws

$$
u_{t}+f(u)_{x}=0 \text {, }
$$

depending on a single space variable $x \in R$ and the time $t>0$, where $u=$ $\left(u_{1}, u_{2}, \ldots, u_{n}\right)^{\mathrm{T}}$ and $f(u)=\left(f_{1}(u), f_{2}(u), \ldots, f_{n}(u)\right)^{\mathrm{T}}$ are $n$-dimensional vectors. A weak solution $u(x, t)$ of $(2.7)$ is known as a (simple) shock wave if it has the form

$$
u(x, t)= \begin{cases}u_{l}, & x<s t, \\ u_{r}, & x>s t .\end{cases}
$$

In that case $u_{l}, u_{r}$ and $s$ are related by the Rankine-Hugoniot jump condition

$$
f\left(u_{l}\right)-f\left(u_{r}\right)=s\left(u_{l}-u_{r}\right) \text {. }
$$


Conversely, if (2.9) holds, then the function $u(x, t)$ defined by $(2.8)$ is indeed a weak solution of (2.7) [20].

It is known that (the weak) solutions of (2.7) with given initial data are not unique. To guarantee uniqueness one imposes an extra condition on solutions of (2.7) known as an entropy condition. Such a condition is supposed to yield the physically relevant solution, namely, the one which is a limit of solution of the corresponding viscous equation:

$$
u_{t}+f(u)_{x}=\mu u_{x x}
$$

$\mu=\operatorname{diag}\left(\mu_{1}, \mu_{2}, \ldots, \mu_{n}\right)>0$, as the viscosity parameters $\mu \rightarrow 0$.

A solution $u_{\mu}(x, t)$ of $(2.10)$ is known as a travelling wave which joins $u_{l}$ to $u_{r}$ if it can be written in the form

$$
u_{\mu}(x, t)=v(x-s t) \text {, }
$$

where the function $v(y),-\infty<y<\infty$, satisfies

$$
\lim _{y \rightarrow-\infty} v(y)=u_{l}, \quad \lim _{y \rightarrow+\infty} v(y)=u_{r} .
$$

If $u_{\mu}(x, t)$ is a travelling wave, then $u_{l}, u_{r}$ and $s$ satisfy the Rankine-Hugoniot equation (2.9) and

$$
\mu \frac{d v}{d y}=f(v)-f\left(u_{l}\right)-s\left(v-u_{l}\right)
$$

Observe that (2.9) shows that $u_{r}$ as well as $u_{l}$ are rest points of the system (2.13). Thus, upon restricting ourselves to travelling waves, the existence of shock wave (2.8) corresponds to the existence of an orbit (trajectory) $v(y)$, of the system (2.13) which joins the rest point $u_{l}$ to the rest point $u_{r}$, and $v(x-s t)$ tends to the shock wave solution $(2.8)$ as $\mu \rightarrow 0$ [20].

Definition 2.1. We say that the shock wave solution (2.8) of the system (2.7) admits structure or possesses structure if the travelling wave solution $v(y)$ exists and

$$
\begin{aligned}
& v(y) \rightarrow u_{l} \quad \text { for } y<0 \\
& v(y) \rightarrow u_{r} \quad \text { for } y>0
\end{aligned}
$$

as $\mu \rightarrow 0$. We also say that the shock wave solution (2.8) has the usual type of profile or the flow is stable if

$$
\begin{aligned}
& v(y) \rightarrow u_{l} \quad \text { for } y<0 \\
& v(y) \rightarrow u_{r} \quad \text { for } y>0
\end{aligned}
$$

uniformly as the maximum of the viscosity parameters $\mu_{1}, \ldots, \mu_{n}$ approaches zero, [11].

Finally, the shock wave $(2.8)$ is also called a shock between $u_{l}$ and $u_{r}$.

Suppose $\delta \varepsilon \neq 0$ and the system (2.1) admits four rest points, namely $u_{0}$, $u_{1}, u_{2}$ and $u_{3}$ (ordered by increasing density). As discussed in [9], [10], [17] and [18], the structure problem reduces to the existence of complete orbits of the system (2.1) joining $u_{i}$ and $u_{j}$ for $i \neq j$ (of course satisfying "stability condition"). It is known that if an orbit runs from $u_{i}$ to $u_{j}$, then $i<j$ [9] (see also Theorem 3.2 in the next section).

Shock waves between $u_{0}$ and $u_{1}$ ( $u_{0}$ should be left and $u_{1}$ right of the shock) are called fast shocks. Shock waves between $u_{2}$ and $u_{3}\left(u_{2}\right.$ should be 
left and $u_{3}$ right of the shock) are called slow shocks. Shock waves between $u_{i}$ and $u_{j}, i=0,1$ and $j=2,3\left(u_{i}\right.$ as left and $u_{j}$ as right of the shock) are called intermediate shocks.

In $\S 3$, by using a technique, similar to those that have been used by Conley and Smoller in [4]-[8], [20], [21] and [22] and the author in [12] and [15], we shall build an isolating neighbourhood containing all of the bounded complete orbits of the system (2.1). In $\S 4$, we shall show that the fast and the slow shocks have structure. In $\S 5$ we will see that none of the intermediate shocks admits structure.

\section{THE ISOLATING NEIGHBOURHOOD}

In this section we shall show that the system (2.1) is gradient-like. This will enable us to prove that the set of all bounded complete orbits of this system is bounded and bounded away from $T=0$ and $V=0$ independent of all of the viscosity parameters. The related "isolating neighbourhood" contains the travelling wave solutions [20] for the fast and the slow shocks. Prior to this we have the following.

Given an autonomous system of ordinary differential equations in $R^{n}$

$$
\frac{d x}{d t}=f(x),
$$

we will denote by $x \cdot t$ the value of the solution of this system at time $t$ that is $x$ initially. As $f$ is assumed smooth, $x \cdot t$ will be uniquely defined for each $x$ on an open interval of $t$ contains 0 .

For $S \subset R^{n}, J \subset R$, we let $S \cdot J=\{x \cdot t: x \in S, t \in J\}$. The set $S$ is called invariant if $S \cdot R=S$. For $Y \subset R^{n}$ the $\omega$-limit set of $Y$ is defined to be the maximal invariant set in the closure of $Y \cdot[0, \infty)$. Similarly the $\alpha$-limit set of $Y$ is defined to be the maximal invariant set in the closure of $Y \cdot(-\infty, 0]$.

By an orbit we mean a solution of (3.1) which is defined on an open interval. By a complete orbit we mean an orbit which is defined for all values of $t \in R$. We say that $\gamma(t)$ is an orbit running from $x_{0}$ to $x_{1}$, if $\gamma(t)$ is a complete orbit and $\lim _{t \rightarrow-\infty} \gamma(t)=x_{0}$ and $\lim _{t \rightarrow+\infty} \gamma(t)=x_{1}$. Then $x_{0}$ and $x_{1}$ must be rest points. Such an orbit is called a heteroclinic orbit.

The system (3.1) is called gradient-like in the open set $U \subset R^{n}$, if there is a continuous real-valued function $h$ on $U$ which is strictly increasing on nonconstant solutions of (3.1). The function $h$ is called the gradient-like function. We also say that this system is gradient-like with respect to $h$ on $U$.

Note that the $\omega$-limit set of the orbit $x \cdot t$ means the set of limit points of sequences $x \cdot t_{n}$ as $t_{n}$ tends to plus infinity, and the $\alpha$-limit set of this orbit is the set of limit points of these sequences as $t_{n}$ goes to minus infinity. It is well known that for bounded complete orbit each of these two sets is nonempty, compact, connected and invariant. In the case of a gradient-like system the restriction of the gradient-like function to each of these sets is constant. Therefore each of them consists of rest points [2].

A bounded open set $N \subset R^{n}$ is called an isolating neighbourhood if $x \in \partial N$ implies $x \cdot R \not \subset \bar{N}$ (i.e., closure of $N$ ). An invariant set $S$ is called an isolated invariant set if $S$ is the maximal invariant set in some isolating neighbourhood $N$. In this case say $N$ is an isolating neighbourhood of $S$.

Observe that isolated invariant sets are closed and that if $N$ is an isolating 
neighbourhood of $S$, then any open set between $N$ and $S$ is also an isolating neighbourhood of $S$. The word isolated refers to the fact that there are no invariant sets between $S$ and $\bar{N}$.

An isolating neighbourhood $B$ is called an isolating block, if for each $x \in \partial B$ there is an $\varepsilon>0$ such that $x \cdot(0, \varepsilon) \cap \bar{B}=\varnothing$ or $x \cdot(-\varepsilon, 0) \cap \bar{B}=\varnothing$. It is well known that every neighbourhood of an isolated invariant set contains one in the form of an isolating block [3] and [20].

Let $B$ be an isolating block for the isolated invariant set $S$. The set $b^{+}=$ $\{x \in \partial B: \exists \varepsilon>0 \mid x \cdot(0, \varepsilon) \cap \bar{B}=\varnothing\}$ is called the exit set of $B$.

Definition 3.1. Let $S$ be an isolated invariant set and $B$ any isolating block of $S$ with the exit set $b^{+}$. We define the Conley index of $S$ by $h(S)=\left[\frac{\bar{B}}{b^{+}}\right]$, i.e., the homotopy equivalence class of the pointed space $\left(\frac{\bar{B}}{b^{+}}, \frac{b^{+}}{b^{+}}\right)$.

It is well known that $h(S)$ is independent of $B$ and only depends on $S$. Namely it is well defined. For more details the reader is referred to [2], [3], [19] and [20].

Now, we shall show that the system $(2.1)$ is gradient-like. To do this, we define the real-valued function $P$ on $V>0, T>0$ by

$$
\begin{aligned}
P(u)=T^{-1}\left\{\frac{1}{2} V^{2}\right. & +\frac{1}{2}\left(V-\delta^{2}\right)\left(B_{2}^{2}+B_{3}^{2}\right)-J V \\
& \left.-\frac{1}{2}\left(y_{2}^{2}+y_{3}^{2}\right)+\varepsilon B_{2}-f(V, T)+C\right\}
\end{aligned}
$$

where $f(V, T)$ is the Helmholtz free energy function [23]. This function satisfies

$$
f_{V}=-p, \quad f_{T}=-S, \quad e=f+T S .
$$

From now on we assume that $\chi=0$ whenever $\alpha=0$. Moreover $\chi \alpha^{-1}$ is bounded from above as $(\alpha, \chi) \rightarrow 0$ and for the case $\alpha=\chi=0, \chi \alpha^{-1}=0$ too. Although from the definitions of $\alpha$ and $\chi$ these assumptions seem to be irrelevant, from the physical point of view these make sense as the Hall effect has not appeared in the MHD shock waves structure equations. See [10] or [16].

Theorem 3.1. The system (2.1) is gradient-like with respect to $P(u)$ in the region $\left\{u \in R^{6}: V, T>0\right\}$ for all choices of the viscosities except for two cases, (i) $\eta=k=\sigma^{-1}=0$, (ii) $\alpha \neq 0, \sigma^{-1}=\varepsilon=0$.

Proof. If we differentiate $P(u)$ along the orbits of the system (2.1), for the case $\alpha \eta k \neq 0$, by using (3.3) we obtain

$$
\begin{aligned}
\frac{d P}{d t}= & T^{-2} G_{6} \dot{T}+T^{-1} G_{5} \dot{V}+T^{-1}\left(G_{3}+\chi \alpha^{-1} \delta G_{2}+\sigma^{-1} \frac{G_{1}}{\alpha V}\right) \dot{B}_{2} \\
& +T^{-1}\left(G_{4}-\chi \alpha^{-1} \delta G_{1}+\sigma^{-1} \frac{G_{2}}{\alpha V}\right) \dot{B}_{3}-T^{-1} G_{1} \dot{y}_{2}-T^{-1} G_{2} \dot{y}_{3} \\
= & \frac{T^{-2}}{k} G_{6}^{2}+\frac{T^{-1}}{\eta} G_{5}^{2}+\sigma^{-1} T^{-1}\left(\dot{B}_{2}^{2}+\dot{B}_{3}^{2}\right)
\end{aligned}
$$

where $G_{i}$ means $G_{i}(u), 1 \leq i \leq 6$. It is obvious that for the cases $\eta=0$ or $k=0$ the related terms drop out, and for the case $\alpha=\chi=0$ we obtain the same value for $\frac{d P}{d t}$. Thus $P(u)$ is nondecreasing along the orbits of the system (2.1). 
Now, suppose there is an orbit of the system (2.1), say $\gamma(t)$, and $t_{1}<t_{2}$ such that $P\left(\gamma\left(t_{1}\right)\right) \geq P\left(\gamma\left(t_{2}\right)\right)$. Then from $\frac{d P}{d t} \geq 0, P(\gamma(t))$ must be constant in the interval $\left(t_{1}, t_{2}\right)$. Hence in this interval $\frac{d P}{d t} \equiv 0$. This implies $G_{6}(\gamma(t)) \equiv$ $G_{5}(\gamma(t)) \equiv \sigma^{-1} \dot{B}_{2}(t) \equiv \sigma^{-1} \dot{B}_{3}(t) \equiv 0$ for $t \in\left(t_{1}, t_{2}\right)$ and all choices of the viscosities, where $\gamma(t)=\left(B_{2}(t), B_{3}(t), y_{2}(t), y_{3}(t), V(t), T(t)\right)^{\mathrm{T}}$.

Here, we consider the following four different cases to show that $\gamma(t)$ must be a rest point.

Case 1. $\eta k \neq 0$. From $G_{5}(\gamma(t)) \equiv G_{6}(\gamma(t)) \equiv 0$, we get $\dot{T}(t) \equiv \dot{V}(t) \equiv 0$. Thus $T(t)$ and $V(t)$ are constants.

If $\sigma^{-1} \neq 0$, then $\dot{B}_{2}(t) \equiv \dot{B}_{3}(t) \equiv 0$. Hence $y_{2}(t) \equiv y_{3}(t) \equiv 0$, and $B_{2}(t)$ and $B_{3}(t)$ are constants.

If $\sigma^{-1}=\alpha=\chi=0$, then the first four equations of the system (2.1) imply $y_{2}(t) \equiv y_{3}(t) \equiv 0$ and $B_{i}(t)$ is constant for $i=2,3$.

Suppose $\sigma^{-1}=0, \alpha \varepsilon \neq 0$. Since $V(t)$ is constant, the first four equations of the system (2.1) are linear with constant coefficients for $B_{2}, B_{3}, y_{2}$ and $y_{3}$. Also from $G_{5}(\gamma(t)) \equiv 0$ we obtain $B_{2}^{2}(t)+B_{3}^{2}(t) \equiv$ const . These imply that

$$
B_{2}(t)=A \cos (\omega t+\theta)+C_{1}, \quad B_{3}(t)=A \sin (\omega t+\theta)+C_{2},
$$

for some constants $\omega, \theta, A, C_{1}$ and $C_{2}$. Hence $y_{2}^{2}(t)+y_{3}^{2}(t) \equiv$ const. Then $G_{6}(\gamma(t)) \equiv 0$ implies $\varepsilon B_{2}(t) \equiv$ const. Thus $B_{i}(t)$ and $y_{i}(t)$ are constants, $i=2,3$.

Case 2. $\eta \neq 0, k=0$. From $G_{5}(\gamma(t)) \equiv 0$, we obtain $\dot{V}(t) \equiv 0$ or $V(t)$ is constant. If $\sigma^{-1} \neq 0$ or $\sigma^{-1}=\alpha=\chi=0$, similar to the above case, we obtain $y_{i}(t)$ and $B_{i}(t)$ are constants. Then $G_{6}(\gamma(t)) \equiv 0$ implies $T(t)$ is constant too.

Suppose $\sigma^{-1}=0$ and $\alpha \varepsilon \neq 0$. Differentiate $G_{6}(\gamma(t)) \equiv 0$ to obtain

$$
\begin{aligned}
e_{T} \dot{T}+ & {\left[e_{V}-V-\frac{1}{2}\left(B_{2}^{2}+B_{3}^{2}\right)+J\right] \dot{V}-\left(V-\delta^{2}\right)\left(B_{2} \dot{B}_{2}+B_{3} \dot{B}_{3}\right) } \\
& +y_{2} \dot{y}_{2}+y_{3} \dot{y}_{3}-\varepsilon \dot{B}_{2} \\
= & e_{T} \dot{T}-\left(V-\delta^{2}\right)\left(\frac{B_{2} y_{2}}{\alpha V}+\frac{B_{3} y_{3}}{\alpha V}\right) \\
& +\frac{y_{2}}{\alpha V}\left[\varepsilon+\left(V-\delta^{2}\right) B_{2}-\chi \alpha^{-1} \delta y_{3}\right] \\
& +\frac{y_{3}}{\alpha V}\left[\left(V-\delta^{2}\right) B_{3}+\delta \chi \alpha^{-1} y_{2}\right]-\varepsilon \frac{y_{2}}{\alpha V}=e_{T} \dot{T} \equiv 0
\end{aligned}
$$

Since $e_{T}=T S_{T}>0, \dot{T}(t) \equiv 0$ or $T(t)$ is constant. Now, similar to Case 1, we can deduce $y_{i}(t)$ and $B_{i}(t)$ are constants, $i=2,3$.

Case 3. $\eta=0, k \neq 0$. Again from $G_{6}(\gamma(t)) \equiv 0$ we obtain $\dot{T}(t) \equiv 0$ or $T(t)$ is constant. If $\sigma^{-1} \neq 0$, then $B_{i}(t)$ is constant for $i=2,3$. This and the first two equations of $(2.1)$ and $G_{5}(\gamma(t)) \equiv G_{6}(\gamma(t)) \equiv 0$ imply $V(t)$ and $y_{i}(t), i=2,3$, are constants. If $\sigma^{-1}=\alpha=\chi=0$, then from the first four equations of the system (2.1) we get $y_{2}(t) \equiv y_{3}(t) \equiv B_{3}(t) \equiv 0$ and $B_{2}(t)=-\varepsilon\left[V(t)-\delta^{2}\right]^{-1}$. Substituting these into $G_{5}(\gamma(t)) \equiv G_{6}(\gamma(t)) \equiv 0$, we conclude that $V(t)$ and $B_{2}(t)$ are constants.

Suppose $\sigma^{-1}=0$ and $\alpha \varepsilon \neq 0$. By differentiating $G_{6}(\gamma(t)) \equiv 0$ and using the identities $e_{V}=T S_{V}-p$ and $\dot{T}(t) \equiv 0$, similar to Case 2, we get $T S_{V} \dot{V}(t) \equiv 0$ 
or $V(t)$ is constant. Now, using an argument similar to Case 1 , we can deduce $B_{i}(t)$ and $y_{i}(t), i=2,3$, are constants too.

Case 4. $\eta=k=0, \sigma^{-1} \neq 0$. From $\sigma^{-1} \neq 0$, we obtain $\dot{B}_{i}(t) \equiv 0, i=2,3$. These and the first two equations of the system (2.1) imply $B_{i}(t)$ and $y_{i}(t)$ are constants. Then from $G_{i}(\gamma(t)) \equiv 0, i=5,6$, we see that $V(t)$ and $T(t)$ are constants too.

According to the above, $\gamma(t)$ must be constant in the interval $\left(t_{1}, t_{2}\right)$ for all values of the viscosities except for the cases: $k=\eta=\sigma^{-1}=0$ and $\alpha \neq 0$, $\sigma^{-1}=\varepsilon=0$. Thus $\gamma(t)$ is a rest point for those cases. Hence $P(u)$ is strictly increasing along any nonconstant orbit of the system (2.1) for the above cases. The proof is complete by the definition.

Remarks. (1) Note that from (3.2) and (3.3) we have

$$
P(u)=T^{-1}\left\{-G_{6}(u)+T S(u)\right\} .
$$

Thus, for the case $k=0, P(u)=S(V, T)$. Because of this fact and the above theorem, $P(u)$ can be called a generalized entropy for the system (2.1).

(2) For the case $k+\eta+\sigma^{-1}=0$, from the proof of Theorem 3.1, we have $\frac{d P}{d t} \equiv 0$, i.e., $P(u)$ is constant along the orbits of the system (2.1). Thus along each complete orbit of this system $S(V, T)$ must be constant. On the other hand from Lemma 2.1, the entropy admits different values at different rest points. This means, in this case, the system (2.1) does not have any heteroclinic orbits. This case was studied by Professor Germain in [9] and [10] and by Peyret for $\chi=0$ in [17] in detail.

(3) The system (2.1) is not gradient-like, when $\sigma^{-1}=\varepsilon=0, \alpha \neq 0$. We will prove this claim by showing that in the case $\alpha \neq 0, \sigma^{-1}=\varepsilon=0$, the system (2.1) has some nonconstant periodic solutions.

Example. Suppose in the system (2.1), $\alpha \neq 0, \sigma^{-1}=\varepsilon=0$. Choose $\bar{V}=$ $\chi \alpha^{-1} \delta+\delta^{2}-1>0$, and let $A>0$ and $\bar{T}>0$ be any solutions of the equations

$$
\begin{aligned}
& A^{2}+p(\bar{V}, \bar{T})+\bar{V}-J=0, \\
& e(\bar{V}, \bar{T})-\frac{1}{2} \bar{V}^{2}-\left(\chi \delta \alpha^{-1}-2\right) A^{2}+J \bar{V}-C=0,
\end{aligned}
$$

which exist if $V_{3}<\bar{V}<V_{0}$. Then the curve

$$
u(t)=\left(B_{2}(t), B_{3}(t), y_{2}(t), y_{3}(t), V(t), T(t)\right)^{\mathrm{T}}
$$

with $B_{2}(t)=y_{3}(t)=A(\cos \omega t+\sin \omega t), B_{3}(t)=-y_{2}(t)=A(\sin \omega t-\cos \omega t)$, $\omega=\alpha^{-1}\left(\delta^{2}+\alpha^{-1} \chi \delta-1\right)^{-1}, V(t)=\bar{V}$ and $T(t)=\bar{T}, t \in R$, is a periodic solution of the system (2.1). Therefore in this case the system (2.1) is not gradient-like.

The next theorem is valid in some sense when the system (2.1) is gradientlike even for the case $\varepsilon=0$. Since, in $\S 2$, the rest points are considered for the case $\varepsilon \neq 0$, we state and prove this theorem for $\varepsilon \neq 0$. As we mentioned before, the case $\varepsilon=0$ is not considered in this paper.

Theorem 3.2. Suppose in the system (2.1), $\varepsilon \neq 0$ and this system is gradient-like with respect to $P(u)$ in $\{u: V>0, T>0\}$. If $\gamma(t)$ is a nonconstant bounded 
complete orbit of this system, then this orbit must be running from a rest point $u_{i}$ to a rest point $u_{j}$ for some $i<j, 0 \leq i, j \leq 3$.

Proof. Since the system (2.1) is gradient-like and the rest points are isolated, $\lim _{t \rightarrow-\infty} \gamma(t)$ and $\lim _{t \rightarrow+\infty} \gamma(t)$ both exist and lie in the rest points set $\left\{u_{m}\right.$ : $0 \leq m \leq 3\}$. On the other hand, from (3.4), $P\left(u_{m}\right)=S\left(u_{m}\right)$. Thus by Lemma $2.1 P\left(u_{i}\right)<P\left(u_{j}\right)$ for $i<j$. Since $P(u)$ is increasing along $\gamma(t)$, this orbit must be running from $u_{i}$ to $u_{j}$ for some $i<j, 0 \leq i, j \leq 3$.

Now we will show that the set of all bounded complete orbits of the system (2.1) is bounded and bounded away from $T=0$ and $V=0$. In fact we shall find our bounds independent of $\lambda=\left(\alpha, \eta, k, \sigma^{-1}, \chi\right)$ and $C$ whenever $\varepsilon, J$ and $\delta$ are fixed.

From now on, without loss of generality we may assume that $\chi \geq 0$. For the case $\chi<0$, the negative sign can be passed to $\delta$ in (2.2).

Let $S(\lambda, C)$ be the set of all points which lie on the complete bounded orbits of the system (2.1) contained in $V, T>0$ corresponding to fixed $\lambda$ and $C$. Let $\lambda_{0}=\left(\alpha_{0}, \eta_{0}, k_{0}, \sigma_{0}^{-1}, \chi_{0}\right)>0$ be given. By Theorem 2.1 , there is a number $C_{0}$ such that for all $0 \leq \lambda \leq \lambda_{0}$ and $C>C_{0}$ the system (2.1) admits no rest point. For $C \ll C_{0}$ it admits four rest points. For $C_{1}<C_{0}$ and $\lambda_{0}$ we define

$$
S^{\prime}=S^{\prime}\left(\lambda_{0}, C_{1}\right)=\bigcup_{I} S(\lambda, C)
$$

where

$$
I=\left\{(\lambda, C): 0 \leq \lambda \leq \lambda_{0}, C_{1} \leq C \leq C_{0}+1, \eta, k>0\right\} .
$$

Note that for each $(\lambda, C) \in I$ a complete orbit is a rest point or a heteroclinic orbit.

The rest points of the system (2.1) are given by (2.4) which are independent of $\lambda$. The following lemma gives some bounds for the fifth and the sixth components of those points of $S^{\prime}$ which are rest points.

Lemma 3.1. There are constants $0<a_{1}<a_{2}<\delta^{2}<a_{3}<a_{4}$ and $0<b_{1}<b_{2}$ such that, if $\bar{u} \in S^{\prime}$ is a rest point of the system (2.1) corresponding to some $(\lambda, C) \in I$, then $(\bar{V}, \bar{T}) \in Y$, where $\bar{V}$ and $\bar{T}$ are the fifth and the sixth components of $\bar{u}$ and

$$
Y=\left\{(V, T): V \in\left[a_{1}, a_{2}\right] \cup\left[a_{3}, a_{4}\right], T \in\left[b_{1}, b_{2}\right]\right\} .
$$

Proof. As we know $(\bar{V}, \bar{T})$ is a solution of equations (2.3) for some $C_{1} \leq C \leq$ $C_{0}$, independent of $\lambda$. From (2.3) we see that $(\bar{V}, \bar{T})$ is a continuous function of $C$ (implicit function theorem). Since, for fixed $C,(\bar{V}, \bar{T}) \in\{(V, T)$ : $\left.V \in\left(0, \delta^{2}\right) \cup\left(\delta^{2},+\infty\right), T \in(0,+\infty)\right\}$ and the set $\left[C_{1}, C_{0}\right]$ is compact such positive numbers $a_{k}$ and $b_{m}, 1 \leq k \leq 4,1 \leq m \leq 2$, must exist.

The following Lemmas 3.2-3.5 are modifications of Lemmas 3.3-3.6 in [15]. Those parts of the proofs which are similar will be referred to [15].

Lemma 3.2. On $S^{\prime}, V \leq J$.

Proof. Let $u \in S^{\prime}$; then $u \in S(\lambda, C)$ for some $(\lambda, C) \in I$. By considering the fifth equation of the system (2.1), the remainder of the proof is the same as the proof of Lemma 3.3 in [15]. 
Lemma 3.3. There is a constant $a>0$ such that on $S^{\prime}, V \geq a$ and $T \geq a$.

Proof. Suppose such an $a>0$ does not exist. Then similar to the proof of Lemma 3.4 in [15], we can show that: There is a sequence of points $\left\{\bar{u}_{j}\right\}$ in $S^{\prime}$ whose $T$ coordinates converge to zero. Moreover for each $j$, the point $\bar{u}_{j}$ lies on a complete orbit of the system (2.1), say $\gamma_{j}(t)$ corresponding to particular values of $\lambda$ and $C$, say $\lambda_{j}$ and $C_{j}$, such that $\dot{T}=0$ at $\bar{u}_{j}$ on this complete orbit. This means that $G_{6}\left(\bar{u}_{j}\right)=0$ and $\bar{T}_{j} \rightarrow 0\left(\bar{T}_{j}\right.$ is the sixth component of $\bar{u}_{j}$ ). If we denote our gradient-like function for the value $C_{j}$ by $P_{j}(u)$, then from (3.4) we get $P_{j}\left(\bar{u}_{j}\right)=S\left(\bar{u}_{j}\right)$. Since $0<\bar{V}_{j} \leq J$ and $\bar{T}_{j} \rightarrow 0$, by hypothesis $H_{4}, S\left(\bar{u}_{j}\right)=S\left(\bar{V}_{j}, \bar{T}_{j}\right) \rightarrow 0$. Hence $P_{j}\left(\bar{u}_{j}\right) \rightarrow 0$.

Now, let $Y$ be the set of points which is given in Lemma 3.1. Since $Y$ is compact and $S(V, T)$ is continuous, $S_{0}=\inf _{(V, T) \in Y} S(V, T)>0$. By Theorem 3.2, $\gamma_{j}(t)$ tends to a rest point, say $u_{m j}$ as $t$ tends to $-\infty$. Then $P_{j}\left(\bar{u}_{j}\right)>P_{j}\left(u_{m j}\right)=S\left(u_{m j}\right) \geq S_{0}>0$. This contradicts $P_{j}\left(\bar{u}_{j}\right) \rightarrow 0$. Hence such an $a>0$ exists.

In the next two lemmas we assume that $\chi=0$. After finishing the proof of Lemma 3.5, we will see that these lemmas are valid for $\chi \neq 0$ too.

Lemma 3.4. There is a constant $M$ such that if $u \in S^{\prime}$ lies on a complete bounded orbit of the system (2.1) corresponding to $\chi=0$, then $\left|B_{2}\right|,\left|B_{3}\right|,\left|y_{2}\right|$ and $\left|y_{3}\right|$ are less than $M$.

Proof. We will consider three different cases as follows.

Case 1. $\alpha=0$. From the first two equations of the system (2.1) we obtain $y_{i} \equiv 0, i=2,3$. Then the last four equations reduce to the following system of ordinary differential equations (assuming $\chi=0$ ):

$$
\begin{aligned}
\sigma^{-1} \dot{B}_{2} & =\varepsilon+\left(V-\delta^{2}\right) B_{2}, \\
\sigma^{-1} \dot{B}_{3} & =\left(V-\delta^{2}\right) B_{3} \\
\eta \dot{V} & =p(V, T)+V+\frac{1}{2}\left(B_{2}^{2}+B_{3}^{2}\right)-J, \\
k \dot{T} & =e(V, T)-\frac{1}{2} V^{2}-\frac{1}{2}\left(V-\delta^{2}\right)\left(B_{2}^{2}+B_{3}^{2}\right)+J V-\varepsilon B_{2}-C .
\end{aligned}
$$

These equations are the same as the system of equations (1.1) in [5] if we let $\mu=$ 0 in the latter system. Conley and Smoller have shown in [5] for fixed $C$, there is an isolating block for this system which contains all of the bounded complete orbits for all parameter values $0 \leq\left(\eta, k, \sigma^{-1}\right) \leq\left(\eta_{0}, k_{0}, \sigma_{0}^{-1}\right)$. Since $C$ is a regular parameter, this isolating block can be modified to another block which contains all of the heteroclinic orbits for $0 \leq\left(\eta, k, \sigma^{-1}\right) \leq\left(\eta_{0}, k_{0}, \sigma_{0}^{-1}\right)$ and $C_{1} \leq C \leq C_{0}$. Thus in this case such an $M$ exists.

Case 2. $\alpha \neq 0$ and small. Let $a_{i}, 1 \leq i \leq 2$, be the same as in Lemma 3.1 and $a$ as in Lemma 3.3. Let $V, C$ and $r$ with $a_{1} \leq V \leq a_{2}, C_{1} \leq C \leq C_{0}$ and $0<r<\frac{1}{2} \min \left(a_{1}, \delta^{2}-a_{2}\right)$ be fixed. Then $N_{r}=\left\{\left(B_{2}, B_{3}, y_{2}, y_{3}\right)\right.$ : 
$\left.\left(B_{2}+\frac{\varepsilon}{V-\delta^{2}}\right)^{2}+B_{3}^{2}+y_{2}^{2}+y_{3}^{2}<r^{2}\right\}$ is an isolating neighbourhood for the system

$$
\begin{aligned}
& a V \frac{d B_{2}}{d t}=y_{2}, \quad \alpha V \frac{d B_{3}}{d t}=y_{3}, \\
& \alpha V \frac{d y_{2}}{d t}=\varepsilon+\left(V-\delta^{2}\right) B_{2}-\frac{\sigma^{-1}}{\alpha V} y_{2}, \\
& \alpha V \frac{d y_{3}}{d t}=\left(V-\delta^{2}\right) B_{3}-\frac{\sigma^{-1}}{\alpha V} y_{3},
\end{aligned}
$$

which can be continued over the region $M=\left\{(V, T): \frac{a}{2}<V<\frac{1}{2}\left(a_{2}+\delta^{2}\right)\right.$, $\left.\frac{a}{2}<T\right\}$. On the other hand, there is an $0<r_{0}<\frac{1}{2} \min \left(a_{1}, \delta^{2}-a_{2}\right)$ such that for each $\left(r_{1}, r_{2}, r_{3}, r_{4}\right)$ with $\sum_{i=1}^{4} r_{i}^{2} \leq r_{0}^{2}$ the system

$$
\begin{aligned}
\eta \dot{V}= & \frac{1}{2}\left[\left(r_{1}-\frac{\varepsilon}{V-\delta^{2}}\right)^{2}+r_{2}^{2}\right]+V-J+p(V, T) \\
k \dot{T}= & e(V, T)-\frac{1}{2} V^{2}-\frac{1}{2}\left(V-\delta^{2}\right)\left[\left(-\frac{\varepsilon}{V-\delta^{2}}+r_{1}\right)^{2}+r_{2}^{2}\right]+J V \\
& +\frac{1}{2}\left(r_{3}^{2}+r_{4}^{2}\right)-\varepsilon\left(-\frac{\varepsilon}{V-\delta^{2}}+r_{1}\right)-C
\end{aligned}
$$

admits two rest points which lie in the set

$$
\bar{N}=\left\{(V, T): \frac{3}{4} a_{1}<V<a_{2}+\frac{1}{4}\left(\delta^{2}-a_{2}\right), \frac{3}{4} a<T<2 b_{2}\right\}
$$

where $b_{2}$ is given in Lemma 3.1. It is known that for given $\eta>0, k>0$ and $\sum_{i=1}^{4} r_{i}^{2} \leq r_{0}^{2}$ there is a unique orbit of (3.6) connecting the above rest points [13, p. 94]. Along this orbit $V$ is decreasing and $T$ is increasing. Thus $\bar{N}$ is an isolating neighbourhood for (3.6) which contains all of the complete orbits between the above rest points for $\eta, k>0$ and all possible values of $r_{i}$, $1 \leq r_{i} \leq 4$. Therefore by Corollary 2.4 in [5] the set

$$
N_{C}=\left\{u:\left(B_{2}, B_{3}, y_{2}, y_{3}\right) \in N_{r_{0}},(V, T) \in \bar{N}\right\}
$$

is an isolating neighbourhood for the system (2.1) whenever $\min (\eta, k) \gg \alpha$. Thus there is a positive number $A_{0}$ such that if $\operatorname{Max}\left(\frac{\alpha}{\eta}, \frac{\alpha}{k}\right) \leq A_{0}$, then the set of all heteroclinic orbits between $u_{2}$ and $u_{3}$ must lie in $N_{C}$. Since $C$ is a regular parameter, $N_{C}$ can be widened to a block, say $B_{1}$ valid for all flows parametrized by $C_{1} \leq C \leq C_{0}$. Moreover $B_{1}$ contains all of the heteroclinic orbits corresponding to the slow shocks. Similarly there is a block $B_{2}$ containing all of the heteroclinic orbits for the fast shocks whenever $C_{1} \leq C \leq C_{0}$ and $\operatorname{Max}\left(\frac{\alpha}{\eta}, \frac{\alpha}{k}\right) \leq A_{0}$. Therefore there is an $M>0$, such that for small values of $\alpha$ all the complete bounded orbits for the fast and the slow shocks in $S^{\prime}$ lie in the set $\left\{u \in R^{6}:|u| \leq M\right\}$.

Case 3. $\alpha>0$ and large. For simplicity we set $t^{\prime}=\alpha^{-1} t, \mu=\alpha^{-1} \sigma^{-1}$ and $\eta^{\prime}=\eta \alpha^{-1}$. Using these and $\chi=0$ the first five equations of the system $(2.1)$ 


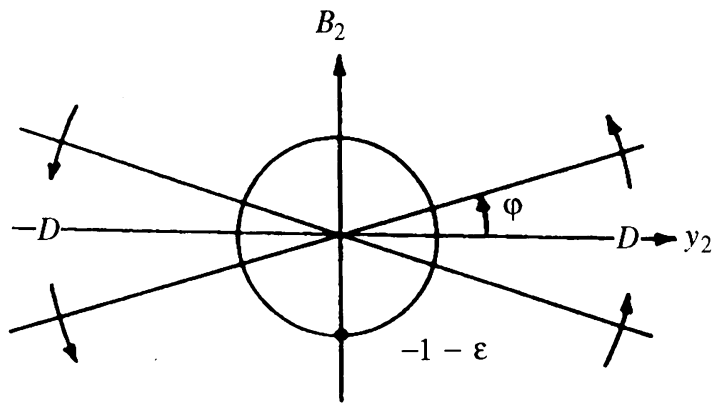

Figure 3.1. The arrows on the boundary of $D$ show the direction of the flow on this set

can be written as

$$
\begin{aligned}
V \frac{d B_{2}}{d t^{\prime}} & =y_{2}, \quad V \frac{d B_{3}}{d t^{\prime}}=y_{3}, \\
V \frac{d y_{2}}{d t^{\prime}} & =\varepsilon+\left(V-\delta^{2}\right) B_{2}-\mu \frac{y_{2}}{V}, \\
V \frac{d y_{3}}{d t^{\prime}} & =\left(V-\delta^{2}\right) B_{3}-\mu \frac{y_{3}}{V}, \\
\eta^{\prime} \frac{d V}{d t^{\prime}} & =\frac{1}{2}\left(B_{2}^{2}+B_{3}^{2}\right)+V-J+p(V, T) .
\end{aligned}
$$

If $a \leq V \leq J$, from the first four equations we see that $\left|B_{i}\right|$ and $\left|y_{i}\right|$, $i=2,3$, decrease at most exponentially. Thus given any positive constants $K$ and $t_{0}^{\prime}$, two constants $M>M_{1}>K$ can be found (depending on the upper bound for $\mu$ ) such that any orbit initiating at point $\left(B_{20}, B_{30}, y_{20}, y_{30}, V_{0}, T_{0}\right)$ with $y_{i 0}^{2} \geq M_{1}$ which is defined for $t^{\prime} \in[0, J]$, either $V \notin[a, J]$ for some $t^{\prime} \in$ $[0, J]$ or $y_{i}^{2}>K$ for all $t^{\prime} \in[0, J], i=2,3$. Moreover any orbit initiating at a point $\left(B_{2}^{\prime}, B_{3}^{\prime}, y_{2}^{\prime}, y_{3}^{\prime}, V^{\prime}, T^{\prime}\right)$ with $\left(B_{i}^{\prime 2}+y_{i}^{\prime 2}\right) \geq M^{2}$ which is defined for $t^{\prime} \in\left[0, t_{0}^{\prime}\right]$, either $V \notin[a, J]$ for some $t^{\prime} \in\left[0, t_{0}^{\prime}\right]$ or $\left(B_{i}^{2}+y_{i}^{2}\right)>2 M_{1}$ for all $t^{\prime} \in\left[0, t_{0}^{\prime}\right], i=2,3$.

Now let $\theta=\tan ^{-1} \frac{B_{2}}{y_{2}}$ with $\theta=0(\bmod 2 \pi)$ on the positive $y_{2}$-axis. A straightforward calculation gives:

$$
\frac{d \theta}{d t^{\prime}}=\frac{1}{V}\left[\cos ^{2} \theta-\frac{\varepsilon}{\left(B_{2}^{2}+y_{2}^{2}\right)^{1 / 2}} \sin \theta-\left(V-\delta^{2}\right) \sin ^{2} \theta+\frac{\mu}{V} \sin \theta \cos \theta\right] .
$$

Thus given $\mu_{0}>0$, we can choose a constant $0<\varphi<\frac{\pi}{4}$ such that for $B_{2}^{2}+y_{2}^{2} \geq$ $(1+\varepsilon)^{2}, a \leq V \leq J, 0 \leq \mu \leq \mu_{0}$ and $|\theta| \leq \varphi$ or $|\theta-\pi| \leq \varphi$, we have $\frac{d \theta}{d t^{\prime}}>\frac{1}{2 J}$. Now define

$$
D=\left\{u: B_{2}^{2} \leq c\left(B_{2}^{2}+y_{2}^{2}\right), a \leq V \leq J, B_{2}^{2}+y_{2}^{2} \geq(1+\varepsilon)^{2}\right\},
$$

where $c=\tan ^{2} \varphi\left(1+\tan ^{2} \varphi\right)^{-1}<\frac{1}{2}$. Thus $D$ depends only on $\mu_{0}$. See Figure 3.1 .

From $\frac{d \theta}{d t^{\prime}}>\frac{1}{2 J}$ we see that any orbit segment on which $V \in[a, J]$ and $B_{2}^{2}+y_{2}^{2} \geq(1+\varepsilon)^{2}$ either stay out of $D$ or else spends a time (i.e., an interval for $\left.t^{\prime}\right)$ in $D$ which is at most $4 \varphi J$. Note that such an orbit having crossed out of any component of $D$, it cannot re-enter the same component of $D$ without passing through the other component of $D$. 
Now for given $\eta_{0}^{\prime}>0$, choose $K>(1+\varepsilon)^{2}$ so large that $c K>2\left(J+\eta_{0}^{\prime}\right)$ and $t_{0}^{\prime}=(2+4 \varphi) J$. Then by the above argument, there are two constants $M>M_{1}>K$ such that any orbit initiating at a point $y_{2}^{2} \geq M_{1}$, either $V \notin$ $[a, J]$ for some $t^{\prime} \in[0, J]$ or $y_{2}^{2}>K$ for all $t^{\prime} \in[0, J]$. Moreover any orbit initiating at a point $B_{2}^{\prime 2}+y^{\prime 2} \geq M^{2}$, either $V \notin[a, J]$ for some $t^{\prime} \in\left[0, t_{0}^{\prime}\right]$ or $B_{2}^{2}+y_{2}^{2}>2 M_{1}>2 K$ for $t^{\prime} \in\left[0, t_{0}^{\prime}\right]$.

We shall now show that $B_{2}^{2}\left(t^{\prime}\right)+y_{2}^{2}\left(t^{\prime}\right)<M^{2}$ on $S^{\prime}$ for $\mu \leq \mu_{0}$ and $\eta^{\prime} \leq \eta_{0}^{\prime}$. Suppose not then there is an orbit in $S^{\prime}$ which starts at $B_{2}^{2}(0)+y_{2}^{2}(0) \geq M^{2}$ and along that orbit $B_{2}^{2}\left(t^{\prime}\right)+y_{2}^{2}\left(t^{\prime}\right)>2 M_{1}>2 K>\frac{4}{c}\left(J+\eta_{0}^{\prime}\right)$ for $t^{\prime} \in\left[0, t_{0}^{\prime}\right]$. Note that in $S^{\prime}, V \notin[a, J]$ cannot occur. If the orbit segment is ever in $D$ it gets out in time $4 \varphi J$. Thus if the orbit does not lie completely out of $D$ for the time interval $[0, J]$, then it goes through a boundary point of $D$ at the time $t_{1}^{\prime} \in[0, J+4 \varphi J]$. Since $B_{2}^{2}\left(t_{1}^{\prime}\right)=c\left[B_{2}^{2}\left(t_{1}^{\prime}\right)+y_{2}^{2}\left(t_{1}^{\prime}\right)\right]$ and $0<c<\frac{1}{2}$, we must have $y_{2}^{2}\left(t_{1}^{\prime}\right) \geq M_{1}$. Thus, by the above argument, in the time interval $\left[t_{1}^{\prime}, t_{1}^{\prime}+J\right]$ along the orbit, $y_{2}^{2}\left(t^{\prime}\right)>K>0$. Since the orbit cannot re-enter $D$ without crossing $y_{2} \equiv 0$, the orbit must lie outside of $D$ for the time interval $\left[t_{1}^{\prime}, J+t_{1}^{\prime}\right]$. Since $\left[t_{1}^{\prime}, J+t_{1}^{\prime}\right] \subset\left[0, t_{0}^{\prime}\right], B_{2}^{2}\left(t^{\prime}\right) \geq c\left[B_{2}^{2}\left(t^{\prime}\right)+y_{2}^{2}\left(t^{\prime}\right)\right] \geq 2\left(J+\eta_{0}^{\prime}\right)$ for a time interval $J$. Therefore for $0<\eta^{\prime} \leq \eta_{0}^{\prime}$

$\eta^{\prime} \frac{d V}{d t^{\prime}}=\frac{1}{2}\left[B_{2}^{2}\left(t^{\prime}\right)+B_{3}^{2}\left(t^{\prime}\right)\right]+V\left(t^{\prime}\right)-J+p\left(V\left(t^{\prime}\right), T\left(t^{\prime}\right)\right) \geq \frac{1}{2} B_{2}^{2}\left(t^{\prime}\right)-J \geq \eta_{0}^{\prime} \geq \eta^{\prime}$ or $\frac{d V}{d t^{\prime}} \geq 1$ for the time interval $\left[t_{2}^{\prime}, t_{2}^{\prime}+J\right]$ where $t_{2}^{\prime}=0$ or $t_{1}^{\prime}$. Thus

$$
\int_{t_{2}^{\prime}}^{t_{2}^{\prime}+J} \frac{d V}{d t^{\prime}} d t^{\prime} \geq J \quad \text { or } \quad V\left(t_{2}^{\prime}+J\right)>J .
$$

Such an orbit cannot lie in $S^{\prime}$. Hence $B_{2}^{2}\left(t^{\prime}\right)+y_{2}^{2}\left(t^{\prime}\right) \leq M^{2}$ on $S^{\prime}$ for $\mu \leq \mu_{0}$ and $0<\eta^{\prime} \leq \eta_{0}^{\prime}$.

With a similar argument for $B_{3}$ and $y_{3}$, the lemma is proved.

Lemma 3.5. There is a constant $T_{0}$ such that if $u \in S^{\prime}$ lies on a bounded complete orbit of the system (2.1) (assuming $\chi=0$ ), then $T \leq T_{0}$ at $u$.

Proof. By considering the sixth equation of the system (2.1), the proof is similar to the proof of Lemma 3.6 in [15].

Now, define the set $N_{0}=N\left(\alpha_{0}, \eta_{0}, k_{0}, \sigma_{0}^{-1}, C_{1}\right)$ by

$$
N_{0}=\left\{u \in R^{6}:|u|<M+J+T_{0}, V>\frac{a}{2}, T>\frac{a}{2}\right\}
$$

where $a, M$ and $T_{0}$ are as in the above lemmas. Then $N_{0}$ is an isolating neighbourhood for all flows parametrized by $(\lambda, C) \in I, \chi=0$. Moreover $N_{0}$ contains all of the bounded complete orbits. Since $\chi$ is a regular parameter, $N_{0}$ can be "widened" to a block valid for all flows parametrized by $(\lambda, C) \in I$, which contains all of the bounded complete orbits. Thus we have proved the following theorem.

Theorem 3.3. Given the set of numbers $J>0, \delta \neq 0, C_{1}, \varepsilon>0$ and $\lambda_{0}=$ $\left(\alpha_{0}, \eta_{0}, k_{0}, \sigma_{0}^{-1}, \chi_{0}\right)>0$, there are positive numbers $M$ and $a$ such that the set $N=\left\{u \in R^{6}:|u|\langle M, V\rangle a, T>a\right\}$ is an isolating neighbourhood for all 
flows parametrized by $(\lambda, C) \in I$. Moreover $N$ contains all bounded complete orbits of the system (2.1) for all values of these parameters.

Remark. In the above theorem the bound $a$ is independent of $\lambda$ and $C$. The bound $M$ is independent of $\lambda$ and $C$ for the heteroclinic orbits corresponding to the fast and the slow shocks. For the heteroclinic orbits corresponding to the intermediate shocks, $M$ depends on the upper bound for $\sigma^{-1} \alpha^{-1}$ and $\eta \alpha^{-1}$.

\section{EXISTENCE OF STRUCTURE FOR FAST AND SLOW SHOCKS}

In this section we shall show that if for given values of $J, C, \delta$ and $\varepsilon$ as before the system (2.1) admits the four rest points $u_{i}, 0 \leq i \leq 3$, then for $\alpha \eta k>0$ and $\chi \sigma^{-1} \geq 0$ there exist a heteroclinic orbit between $u_{0}$ and $u_{1}$ and another one between $u_{2}$ and $u_{3}$. Then we will show that, in fact, the related shock waves admits structure [20].

Let $u_{i}, 0 \leq i \leq 3$, be the rest points as before. As we proved in Lemma 2.1, $S\left(u_{i}\right)<S\left(u_{i+1}\right)$ for $0 \leq i \leq 2$. Let $K_{0}=\frac{1}{2}\left[S\left(u_{1}\right)+S\left(u_{2}\right)\right]$, and define

$$
N_{01}=N \cap\left\{u \in R^{6}: P(u)<K_{0}\right\}, \quad N_{23}=N \cap\left\{u \in R^{6}: P(u)>K_{0}\right\}
$$

where $N$ is introduced in Theorem 3.3 and $P(u)$ is defined by (3.2). Let $S_{01}$ and $S_{23}$ be the set of all points on the complete orbits which are contained in $N_{01}$ and $N_{23}$, respectively.

Lemma 4.1. The sets $S_{01}$ and $S_{23}$ are both isolated invariant sets. Moreover $N_{01}$ and $N_{23}$ are isolating neighbourhoods of $S_{01}$ and $S_{23}$, respectively.

Proof. We prove the lemma for $S_{01}$ and $N_{01}$; the proof for the other case is similar. Since $S_{01} \subset S^{\prime}$ and $S^{\prime}$ has no limit point in $\partial N, S_{01}$ cannot have a limit point in $\partial N_{01} \cap \partial N$.

Now, let $\bar{u} \in \partial N_{01} \cap\left\{u: P(u)=K_{0}\right\}$. By Theorem 3.1, $P(\bar{u} \cdot t)$ is an increasing function with respect to $t$. Moreover, it is continuous with respect to $(\bar{u}, t)$. Thus that point as well as a neighbourhood of it leaves the set $\bar{N}_{01}$ as $t$ increases. Hence no point of $S_{01}$ lies in this neighbourhood. Therefore $S_{01} \subset N_{01}$. This means that $S_{01}$ is an isolated invariant set and $N_{01}$ is an isolating neighbourhood of it.

Now, note that $N_{01}$ and $N_{23}$ are also isolating neighbourhoods for all nearby flow generated by parameter value $C$ (in fact for all regular parameters). By Theorem 2.1, there is no rest point for the system (2.1) for $C>C_{0}$. Thus $h\left(S_{01}\right)=h\left(S_{23}\right) \equiv \overline{0}$. On the other hand $u_{0}, u_{1} \in S_{01}$ and $u_{2}, u_{3} \in S_{23}$, also by Theorem 2.4 all of these rest points are nondegenerate. Moreover, the system (2.1) is gradient-like with respect to $P(u)$ in $V, T>0$. Therefore by considering Theorem 22.33 in [20] we have proved the following theorem.

Theorem 4.1. Assume that hypotheses $H_{1}-H_{5}$ hold. Let the system (2.1) admit the four rest points $u_{i}, 0 \leq i \leq 3$, and $\alpha \eta k>0$ and $\chi \sigma^{-1} \geq 0$. Then there is a complete orbit of this system which is running from $u_{0}$ to $u_{1}$ and another one which is running from $u_{2}$ to $u_{3}$.

Now we shall examine the behaviour of the connecting orbits between $u_{i}$ and $u_{i+1}, i=0,2$, whenever $C, \varepsilon, \delta$ and $J$ are fixed and the maximum of the viscosity parameters tends to zero. From this we deduce that both the fast 
and the slow shocks possess structure. We do this for the fast shocks; the same argument works for the slow shocks too.

Consider the set $N_{01}$ which contains the connecting orbits between $u_{0}$ and $u_{1}$ for all values of $\lambda, 0<\lambda \leq \lambda_{0}$, with $\alpha \eta k>0$. For given small $r>0$, let $c_{0}^{r}$ and $c_{1}^{r}$ denote closed balls of radius $r$ about $u_{0}$ and $u_{1}$ respectively, which are contained in the interior of $N_{01}$. Let $N_{r}=N_{01}-\left(c_{0}^{r} \cup c_{1}^{r}\right)$. If the connecting orbit is $u=\gamma(t)$, we denote by $u_{0}^{r}$ and $u_{1}^{r}$ two points of the orbit which meet $\partial c_{0}^{r}$ and $\partial c_{1}^{r}$ respectively, say $\gamma\left(t_{i}\right)=u_{i}^{r}, i=0,1$ (where $t_{i}$ depends on $r$ ). We may assume that for $t_{0}<t<t_{1}, \gamma(t)$ lies in $N_{r}$, for $t<t_{0}$ lies in $c_{0}^{r}$ and for $t>t_{1}$ lies in $c_{1}^{r}$.

Let $P(u)$ be given by (3.2). Let the constants $a, M$ and the isolating neighbourhood $N$ be as in Theorem 3.3. We define the real-valued function $Q$ on $N$ by

$$
Q(u)=P(u)+b\left[\varepsilon y_{2}+\left(V-\delta^{2}\right)\left(B_{2} y_{2}+B_{3} y_{3}\right)\right]
$$

where $b$ is a positive constant which will be introduced later.

If we differentiate $Q$ along the orbits of the system (2.1) we obtain

$$
\begin{aligned}
\frac{d Q}{d t}= & \frac{T^{-2}}{k} G_{6}^{2}+\frac{T^{-1}}{2 \eta}\left[\left(G_{5}+b T B_{2} G_{1}\right)^{2}+\left(G_{5}+b T B_{3} G_{2}\right)^{2}\right] \\
+ & \frac{b}{2 \alpha V}\left[\left(G_{3}+\frac{\sigma^{-1}}{\alpha V} G_{1}+\frac{\chi \delta}{\alpha} G_{2}\right)^{2}+\left(G_{4}+\frac{\sigma^{-1}}{\alpha V} G_{2}-\frac{\chi \delta}{\alpha} G_{1}\right)^{2}\right. \\
& \left.\quad+\left(\frac{\sigma^{-1}}{\alpha V} G_{1}-\frac{\chi \delta}{\alpha} G_{2}\right)^{2}+\left(\frac{\sigma^{-1}}{\alpha V} G_{2}+\frac{\chi \delta}{\alpha} G_{1}\right)^{2}+G_{3}^{2}+G_{4}^{2}\right] \\
+ & \frac{1}{\alpha}\left(\frac{\sigma^{-1} T^{-1}}{2 \alpha V^{2}}+b\right)\left(G_{1}^{2}+G_{2}^{2}\right) \\
+ & \frac{1}{2 \alpha V}\left(\frac{\sigma^{-1} T^{-1}}{\alpha V}-2 \delta^{2} b-2 b \frac{\sigma^{-2}}{\alpha^{2} V^{2}}-2 b \delta^{2} \frac{\chi^{2}}{\alpha^{2}}-\frac{\alpha}{\eta} T V b^{2} B_{2}^{2}\right) G_{1}^{2} \\
+ & \frac{1}{2 \alpha V}\left(\frac{\sigma^{-1} T^{-1}}{\alpha V}-2 \delta^{2} b-2 b \frac{\sigma^{-2}}{\alpha^{2} V^{2}}-2 b \delta^{2} \frac{\chi^{2}}{\alpha^{2}}-\frac{\alpha}{\eta} T V b^{2} B_{3}^{2}\right) G_{2}^{2},
\end{aligned}
$$

where $G_{i}$ means $G_{i}(u), 1 \leq i \leq 6$.

Now suppose $\rho=\max \left(\alpha, \eta, k, \sigma^{-1}, \chi\right) \rightarrow 0$ such that

$$
\frac{1}{m} \leq \frac{\sigma^{-1}}{\alpha} \leq m, \quad \frac{\chi}{\alpha} \leq m, \quad \frac{\alpha}{\eta} \leq m
$$

for some positive constant $m$. Choose $b=\frac{1}{10} a(m+1)^{-3}(M+1)^{-4}$. Then on $N$, for $i=2,3$, we have

$$
\frac{\sigma^{-1} T^{-1}}{\alpha V}-2 \delta^{2} b-2 b \frac{\sigma^{-2}}{\alpha^{2} V^{2}}-2 b \delta^{2} \frac{\chi^{2}}{\alpha^{2}}-\frac{\alpha}{\eta} T V b^{2} B_{i}^{2} \geq 0
$$


Consequently, along the orbits in $N$, we must have

$$
\begin{aligned}
& \frac{d Q}{d t} \geq \frac{1}{\rho}\left\{M^{-2} G_{6}^{2}+\right. \frac{1}{2} M^{-1}\left[\left(G_{5}+b T B_{2} G_{1}\right)^{2}+\left(G_{5}+b T B_{3} G_{2}\right)^{2}\right] \\
&+\frac{1}{2} b M^{-1}\left[\left(G_{3}+\frac{\sigma^{-1}}{\alpha V} G_{1}+\frac{\delta \chi}{\alpha} G_{2}\right)^{2}+\left(G_{4}+\frac{\sigma^{-1}}{\alpha V} G_{2}-\frac{\delta \chi}{\alpha} G_{1}\right)^{2}\right. \\
&+\left(\frac{\sigma^{-1}}{\alpha V} G_{1}-\frac{\chi \delta}{\alpha} G_{2}\right)^{2}+\left(\frac{\sigma^{-1}}{\alpha V} G_{2}+\frac{\chi \delta}{\alpha} G_{1}\right)^{2} \\
&\left.+\left(\frac{1}{2} M^{-3} m^{-1}+b\right)\left(G_{1}^{2}+G_{2}^{2}\right)\right\} .
\end{aligned}
$$

Thus, there is a constant $\delta_{r}>0$ such that along the orbits in $N_{r}, \frac{d Q}{d t} \geq \frac{1}{\rho} \delta_{r}$. Since along the connecting orbit, $\frac{d Q}{d t}>0$,

$$
\begin{aligned}
S\left(u_{1}\right)-S\left(u_{0}\right) & =P\left(u_{1}\right)-P\left(u_{0}\right)=Q\left(u_{1}\right)-Q\left(u_{0}\right) \geq Q\left(u_{1}^{r}\right)-Q\left(u_{2}^{r}\right) \\
& \geq \int_{t_{0}}^{t_{1}} \dot{Q} d t \geq \int_{t_{0}}^{t_{1}} \frac{1}{\rho} \delta_{r} d t=\frac{1}{\rho} \delta_{r}\left(t_{1}-t_{0}\right),
\end{aligned}
$$

so that $\left(t_{1}-t_{0}\right) \leq$ const $\rho$. Therefore the "time". that the orbit is outside of $c_{0}^{r} \cup c_{1}^{r}$ goes to zero as $\rho \rightarrow 0$. This implies that the fast shock (similarly the slow shock) has the usual type of profile or is stable or admits structure. Therefore we have proved the following theorem.

Theorem 4.2. Assume that hypotheses $H_{1}-H_{5}$ hold. Then fast and slow MFD shocks of arbitrary strength for rectilinear motion in the model of plasma have structure.

\section{THE INTERMEDIATE SHOCKS}

As we mentioned earlier, by an intermediate shock, we mean a shock which on one side takes a value in the set $\left\{u_{0}, u_{1}\right\}$ while, on the other side, takes a value in the set $\left\{u_{2}, u_{3}\right\}$. We shall show that none of these shocks has structure. We shall confine our discussion to the intermediate shock between $u_{1}$ and $u_{2}$.

Suppose the shock between $u_{1}$ and $u_{2}$ admits structure. Then there are positive constants $\alpha_{0}, \eta_{0}, k_{0}, \sigma_{0}^{-1}$ and $\chi_{0}$ such that for each

$$
0<\left(\alpha, \eta, k, \sigma^{-1}, \chi\right) \leq\left(\alpha_{0}, \eta_{0}, k_{0}, \sigma_{0}^{-1}, \chi_{0}\right)
$$

a heteroclinic orbit between $u_{1}$ and $u_{2}$ exists. Moreover, there is a constant $M$ such that $|u| \leq M$ whenever $u$ lies on any of these heteroclinic orbits.

Let $M \geq J+1$ and the positive constant $a$ be as in Lemma 3.3. Consider the real-valued function $Q(u)$ which is defined by (4.1) on the region $W=$ $\left\{u \in R^{6}:|u| \leq M, V \geq a, T \geq a\right\}$. Set $b=\frac{a^{2}}{10 M^{2}} \frac{\alpha}{\sigma^{-1}}$ in $Q(u)$. Then for the viscosity parameters $\alpha, \eta, \sigma^{-1}, k>0, \chi \geq 0$ with $\eta \geq M^{2} \delta^{-4} \sigma^{-1}$, $\sigma^{-1} \geq|\delta| a \alpha$ and $\sigma^{-1} \geq|\delta| a \chi$ the inequality (4.3) holds on $W$. Thus by (4.2) 
$Q(u)$ is increasing along the nonconstant orbits of the system $(2.1)$ in $W$ for the above viscosity parameters. Note that by Lemma 3.3 along every heteroclinic orbit we must have $V \geq a$ and $T \geq a$.

Choose the constant $m_{0}$ such that both $0<2 m_{0}<\delta^{2}-V_{2}$ and $m_{0}<\varepsilon / 4 M$ hold, where $V_{2}$ is the fifth component of $u_{2}$. If $\delta^{2}-2 m_{0} \leq V \leq \delta^{2}-m_{0}$, then from (2.2) we have

$$
\left|G_{3}+\frac{\chi \delta}{\alpha} G_{2}+\frac{\sigma^{-1}}{\alpha V} G_{1}\right|=\left|\varepsilon-\left(V-\delta^{2}\right) B_{2}\right| \geq \varepsilon-\left|V-\delta^{2}\right|\left|B_{2}\right| \geq \frac{\varepsilon}{2} .
$$

Now suppose $\gamma(t)$ is a connecting orbit between $u_{1}$ and $u_{2}$ corresponding to the above viscosity parameters lying in $W$. Then it must have a segment on the strip $\delta^{2}-2 m_{0} \leq V \leq \delta^{2}-m_{0}$ which runs from $\delta^{2}-V=m_{0}$ to $\delta^{2}-V=2 m_{0}$ in the time interval $\left[t_{1}, t_{2}\right]$. Then, by considering (4.2), in this interval we must have

$$
\frac{d Q}{d t} \geq \frac{1}{2 \alpha} b M^{-1}\left(\frac{1}{4} \varepsilon^{2}\right)=\frac{1}{8 \alpha} b M^{-1} \varepsilon^{2} .
$$

Thus

$$
S\left(u_{2}\right)-S\left(u_{1}\right)=Q\left(u_{2}\right)-Q\left(u_{1}\right) \geq \int_{t_{1}}^{t_{2}} \frac{1}{8 \alpha} b M^{-1} \varepsilon^{2} d t=\frac{1}{8 \alpha} b M^{-1} \varepsilon^{2}\left(t_{2}-t_{1}\right) .
$$

Now let $M_{0}=\operatorname{Max}_{u \in W}\left|G_{5}(u)\right|$. Then

$$
\begin{aligned}
m_{0} & =\left(\delta^{2}-m_{0}\right)-\left(\delta^{2}-2 m_{0}\right)=\int_{t_{1}}^{t_{2}} \dot{V} d t=\int_{t_{1}}^{t_{2}} \frac{G_{5}(u)}{\eta} d t \\
& \leq \int_{t_{1}}^{t_{2}} \frac{M_{0}}{\eta} d t=\frac{M_{0}}{\eta}\left(t_{2}-t_{1}\right) .
\end{aligned}
$$

Hence

$$
\begin{aligned}
S\left(u_{2}\right)-S\left(u_{1}\right) & \geq \frac{1}{8 \alpha} b M^{-1} \varepsilon^{2}\left(t_{2}-t_{1}\right) \geq \frac{1}{8} b \varepsilon^{2} m_{0} M^{-1} M_{0}^{-1} \frac{\eta}{\alpha} \\
& =\frac{1}{80} m_{0} \varepsilon^{2} a^{2} M^{-3} M_{0}^{-1} \frac{\eta}{\sigma^{-1}},
\end{aligned}
$$

which is impossible if $\frac{\eta}{\sigma^{-1}}$ is large. Therefore we have proved the following theorem.

Theorem 5.1. Given positive numbers $M$ and $a$, then there is positive number $A$ such that for all viscosity parameters $\lambda=\left(\alpha, \eta, k, \sigma^{-1}, \chi\right)>0$ with $\sigma^{-1} \geq$ $|\delta| a \operatorname{Max}(\alpha, \chi)$ and $\eta \geq A \sigma^{-1}$, there is no connecting orbit between $u_{i}$ and $u_{j}$, $i=0,1, j=2,3$, inside the region $\{u:|u| \leq M\}$.

Corollary 5.1. The intermediate MFD shock waves in the model of plasma do not admit structure.

\section{SingulaR VISCOSITIES}

In this section we shall examine the behaviour of the connecting orbits for the fast and the slow shocks as some of the viscosity parameters tend to zero. In particular, we shall see how the general results of the MHD theory [4], [11] and [13] can be obtained when making $(\alpha, \chi) \rightarrow 0$. 
Let $X$ be a topological space and $G$ an infinite collection of subsets of $X$, not necessarily different. The set of all points $x$ of the space $X$ such that every neighbourhood of $x$ contains points of infinitely many sets of $G$ is called the limit superior of $G$ and is denoted by $\overline{\lim } G$ or $\lim \sup G$ [24].

Let $X$ and $Y$ be two topological spaces, $I \subset Y$ and the function $G$ assign to each point $\mu$ of $I$ a subset of $X$, say $G_{\mu}$. For $\mu_{0} \in \partial I$, when $\mu \rightarrow \mu_{0}$, we define $\lim \sup G_{\mu}=\varlimsup \lim G_{\mu}=\bigcup_{J} \varlimsup \overline{\lim } G_{\mu_{k}}$, where $J$ is the set of all sequences in $I$ which converge to $\mu_{0}$.

Let $\Omega_{1} \subset R^{n}, \Omega_{2} \subset R^{m}$ and $\Lambda \subset R_{+}^{\ell}\left(R_{+}=(0, \infty)\right)$ be open with $0 \in \bar{\Lambda}$. For $(x, y) \in \Omega_{1} \times \Omega_{2}$ we consider the system of equations

$$
\dot{x}=f(x, y, \mu), \quad B(\mu) \dot{y}=g(x, y, \mu)
$$

where $\mu \in \Lambda$ is a vector-valued parameter, $B(\mu)$ an $m \times m$ nonsingular diagonal matrix, $B(\mu) \rightarrow 0$ as $\mu \rightarrow 0$, and $f$ and $g$ are continuous on $\Omega_{1} \times \Omega_{2} \times \Lambda$. Now we have the following lemma.

Lemma 6.1. Suppose for each $\mu \in \Lambda$, the system (6.1) has exactly two rest points $\left(x_{0}, y_{0}\right) \neq\left(x_{1}, y_{1}\right)$, independent of $\mu$, in the closure of a bounded domain $\Omega$ with $\bar{\Omega} \subset \Omega_{1} \times \Omega_{2}$. Moreover the following conditions hold.

$C_{1}: f$ is uniformly Lipschitz in $\Omega \times \Lambda$.

$C_{2}$ : For each $\mu \in \Lambda$ there is a heteroclinic orbit of $(6.1)$, say $\gamma(t, \mu)=$ $(x(t, \mu), y(t, \mu))$, running from $\left(x_{0}, y_{0}\right)$ to $\left(x_{1}, y_{1}\right)$, lying in $\Omega$.

$C_{3}$ : There is a continuous function $\bar{f}(x, y)$ from $\bar{\Omega}$ to $R^{n}$ such that $f(x, y, \mu) \rightarrow \bar{f}(x, y)$ uniformly on $\bar{\Omega}$ as $\mu \rightarrow 0$.

$C_{4}: g(\gamma(t, \mu), \mu) \rightarrow 0$ uniformly on any compact interval as $\mu \rightarrow 0$.

$C_{5}$ : Given positive constant a there is a positive constant $M$ such that for $|t| \leq a$ and $|\mu|$ and $\left|\mu^{\prime}\right|$ small,

$\left|y(t, \mu)-y\left(t, \mu^{\prime}\right)\right| \leq M\left[\left|\gamma(0, \mu)-\gamma\left(0, \mu^{\prime}\right)\right|+F\left(t, \mu, \mu^{\prime}\right)+\left|\int_{0}^{t} F\left(s, \mu, \mu^{\prime}\right) d s\right|\right]$ where

$$
F\left(t, \mu, \mu^{\prime}\right)=\left|x(t, \mu)-x\left(t, \mu^{\prime}\right)\right|+\left|g(\gamma(t, \mu), \mu)-g\left(\gamma\left(t, \mu^{\prime}\right), \mu^{\prime}\right)\right| .
$$

$C_{6}$ : There is a continuous function $\bar{g}(x, y, \dot{y})$ from $\Omega_{1} \times \Omega_{2} \times R^{m}$ to $R^{m}$ such that if $\mu_{k} \rightarrow 0$ and $\gamma\left(t, \mu_{k}\right) \rightarrow \gamma(t)=(x(t), y(t))$, uniformly on compact intervals, then $g\left(\gamma\left(t, \mu_{k}\right), \mu_{k}\right) \rightarrow \bar{g}(x(t), y(t), \dot{y}(t))$.

$C_{7}$ : The system

$$
\dot{x}=\bar{f}(x, y), \quad 0=\bar{g}(x, y, \dot{y})
$$

is gradient-like with respect to a function $h$ on $\Omega$ with $h\left(x_{0}, y_{0}\right)<h\left(x_{1}, y_{1}\right)$.

Then $S=\lim \sup \{\gamma(t, \mu): t \in R\} \backslash\left\{\left(x_{i}, y_{i}\right), i=0,1\right\}$ is a nonempty union of heteroclinic orbits of the system (6.2) running from $\left(x_{0}, y_{0}\right)$ to $\left(x_{1}, y_{1}\right)$ in $\bar{\Omega}$.

Proof. Let $\left\{\mu_{k}\right\}$ be a sequence in $\Lambda$ which tends to zero and $\gamma_{k}(t)=\left(x_{k}(t)\right.$, $\left.y_{k}(t)\right)=\left(x\left(t, \mu_{k}\right), y\left(t, \mu_{k}\right)\right)$ be a heteroclinic orbit corresponding to $\mu_{k}$. Since $\bar{\Omega}$ is compact, we may assume that $\left\{\gamma_{k}(0)\right\}$ converges to a point in $\bar{\Omega}-$ $\left\{\left(x_{i}, y_{i}\right), i=0,1\right\}$. Let $a>0$ be given.

Condition $C_{1}$ implies there is a constant $M_{1}$ such that for $|t| \leq a$,

$$
\left|f\left(\gamma_{i}(t), \mu_{i}\right)-f\left(\gamma_{j}(t), \mu_{j}\right)\right|<M_{1}\left[\left|\gamma_{i}(t)-\gamma_{j}(t)\right|+\left|\mu_{i}-\mu_{j}\right|\right] .
$$


Now, set $z_{k}(t)=g\left(\gamma_{k}(t), \mu_{k}\right)$. Then by $C_{4}, z_{k}(t) \rightarrow 0$ uniformly on $[-a, a]$. Moreover by $C_{5}$,

$$
\begin{array}{r}
\left|y_{i}(t)-y_{j}(t)\right| \leq M\left\{\left|\gamma_{i}(0)-\gamma_{j}(0)\right|+\left|x_{i}(t)-x_{j}(t)\right|+\left|z_{i}(t)-z_{j}(t)\right|\right. \\
\left.+\left|\int_{0}^{t}\left[\left|x_{i}(s)-z_{j}(s)\right|+\left|z_{i}(s)-z_{j}(s)\right|\right] d s\right|\right\} .
\end{array}
$$

Thus for $t \in[0, a]$ we can write

$$
\begin{aligned}
\mid x_{i}(t) & -x_{j}(t)|\leq| x_{i}(0)-x_{j}(0)\left|+\int_{0}^{t}\right| f\left(\gamma_{i}(s), \mu_{i}\right)-f\left(\gamma_{j}(s), \mu_{j}\right) \mid d s \\
\leq & \left|\gamma_{i}(0)-\gamma_{j}(0)\right|+M_{1} \int_{0}^{t}\left[\left|x_{i}(s)-x_{j}(s)\right|+\left|y_{i}(s)-y_{j}(s)\right|+\left|\mu_{i}-\mu_{j}\right|\right] d s \\
\leq & \left(1+a M M_{1}\right)\left|\gamma_{i}(0)-\gamma_{j}(0)\right|+M M_{1} a(1+a) H_{i j}+a M_{1}\left|\mu_{i}-\mu_{j}\right| \\
& +M_{1}(1+M(1+a)) \int_{0}^{t}\left|x_{i}(s)-x_{j}(s)\right| d s
\end{aligned}
$$

where $H_{i j}=\sup _{|t| \leq a}\left|z_{i}(t)-z_{j}(t)\right|$. Thus by Gronwall's inequality

$$
\left|x_{i}(t)-x_{j}(t)\right| \leq b\left[\left|\gamma_{i}(0)-\gamma_{j}(0)\right|+\left|\mu_{i}-\mu_{j}\right|+H_{i j}\right] e^{b a}
$$

where $b=1+M_{1}\left[(1+a)(1+M)+M a^{2}\right]$. Since $z_{k}(t) \rightarrow 0$ uniformly on $[0, a]$ $H_{i j} \rightarrow 0$ as $\min (i, j) \rightarrow \infty$. Hence $\left\{x_{k}(t)\right\}$ is uniformly Cauchy on $[0, a]$. On the other hand, by using (6.3), for $t \in[0, a]$ we have

$$
\begin{aligned}
\left|\dot{x}_{i}(t)-\dot{x}_{j}(t)\right| & =\left|f\left(\gamma_{i}(t), \mu_{i}\right)-f\left(\gamma_{j}(t), \mu_{j}\right)\right| \leq M_{1}\left[\left|\gamma_{i}(t)-\gamma_{j}(t)\right|+\left|\mu_{i}-\mu_{j}\right|\right] \\
& \leq M_{1}\left[M\left|\gamma_{i}(0)-\gamma_{j}(0)\right|+(1+a) M H_{i j}+(1+M+M a) G_{i j}\right]
\end{aligned}
$$

where $G_{i j}=\sup _{|t| \leq a}\left|x_{i}(t)-x_{j}(t)\right|$. Hence $\left\{\dot{x}_{k}(t)\right\}$ is uniformly Cauchy on $[0, a]$ too. Finally from (6.3) we see that $\left\{y_{k}(t)\right\}$ and so $\left\{\gamma_{k}(t)\right\}$ are uniformly Cauchy on $[0, a]$. Suppose $\gamma_{k}(t) \rightarrow \gamma(t)=(x(t), y(t))$. Then $y(t)$ is continuous and $\dot{x}_{k}(t) \rightarrow \dot{x}(t)$ uniformly on $[0, a]$. Thus we can write

$$
\dot{x}(t)=\lim _{k \rightarrow \infty} f\left(\gamma_{k}(t), \mu_{k}\right)=\bar{f}(\gamma(t))=\bar{f}(x(t), y(t)),
$$

where in the second equality we used $C_{1}$ as well as $C_{3}$. Also from $C_{4}$ and $C_{6}$

$$
0=\lim _{k \rightarrow \infty} g\left(\gamma_{k}(t), \mu_{k}\right)=\bar{g}(x(t), y(t), \dot{y}(t)) \text {. }
$$

Thus $\gamma(t)$ is a solution of $(6.2)$ in $[0, a)$. Similarly, it is in $(-a, 0]$. Therefore it must be a solution of (6.2) in $R$.

Since the system (6.2) is gradient-like with respect to $h, \gamma(t)$ must be running from $\left(x_{0}, y_{0}\right)$ to $\left(x_{1}, y_{1}\right)$.

Now, if $(\bar{x}, \bar{y}) \in S$, then there are $\left\{\mu_{k}\right\}$ and $\left\{\gamma\left(t, \mu_{k}\right)\right\}$ such that $\mu_{k} \rightarrow 0$ and $\gamma\left(0, \mu_{k}\right) \rightarrow(\bar{x}, \bar{y})$. Thus by the above argument $(\bar{x}, \bar{y})$ lies on a heteroclinic orbit of the system (6.2). This completes the proof.

Lemma 6.2. Suppose in the system (2.1) $\eta, k, \sigma^{-1}>0$ are fixed, $\alpha>0$ and $m \alpha \geq \chi$ for some constant $m>0$. Then there exist positive constants $m_{0}$ and 
$m_{1}>1$ such that $\left|\delta^{2}-V\right| \geq m_{0}$ if $u$ lies on a complete orbit of the system (2.1) running from $u_{0}$ to $u_{1}$ (resp. $u_{2}$ to $u_{3}$ ) whenever $\alpha$ is small and $\eta \geq m_{1} \sigma^{-1}$. Proof. Let the constants $M$ and $a$ and the isolating neighbourhood $N$ be the same as in Theorem 3.3. For $\alpha \leq[a(M+1)(m+1)]^{-1}, b=\frac{a^{2}}{10 M^{2}} \frac{\alpha}{\sigma^{-1}}$ define the real-valued function $Q(u)$ on $N$ by (4.1). Choose the constant $m_{0}$ such that both $0<2 m_{0}<V_{1}-\delta^{2}$ and $m_{0}<\varepsilon / 4 M$ hold, where $V_{1}$ is the fifth component of $u_{1}$. If $\delta^{2}+m_{0} \leq V \leq \delta^{2}+2 m_{0}$, then from (2.2) we have

$$
\left|G_{3}+\frac{\chi \delta}{\alpha} G_{2}+\frac{\sigma^{-1}}{\alpha V} G_{1}\right|=\left|\varepsilon-\left(V-\delta^{2}\right) B_{2}\right| \geq \varepsilon-\left|V-\delta^{2}\right|\left|B_{2}\right| \geq \frac{\varepsilon}{2} .
$$

Now suppose $\gamma(t)$ is a heteroclinic orbit between $u_{0}$ and $u_{1}$ corresponding to the above viscosities having some points in the region $\left|\delta^{2}-V\right| \leq m_{0}$. Then it must have a segment on the strip $\delta^{2}+m_{0} \leq V \leq \delta^{2}+2 m_{0}$ which runs from $V-\delta^{2}=m_{0}$ to $V-\delta^{2}=2 m_{0}$ in the time interval $\left[t_{1}, t_{2}\right]$. In this interval from (4.2) we get

$$
\frac{d Q}{d t} \geq \frac{1}{2 \alpha} b M^{-1}\left(\frac{1}{4} \varepsilon^{2}\right)=\frac{1}{8 \alpha} b M^{-1} \varepsilon^{2}
$$

Thus

$$
\begin{gathered}
S\left(u_{1}\right)-S\left(u_{0}\right)=Q\left(u_{1}\right)-Q\left(u_{0}\right) \geq \int_{t_{1}}^{t_{2}} \frac{b M^{-1} \varepsilon^{2}}{8 \alpha} d t=\frac{b M^{-1} \varepsilon^{2}}{8 \alpha}\left(t_{2}-t_{1}\right), \\
m_{0}=\left(\delta^{2}+2 m_{0}\right)-\left(\delta^{2}+m_{0}\right)=\int_{t_{1}}^{t_{2}} \dot{V} d t=\int_{t_{1}}^{t_{2}} \frac{G_{5}(u)}{\eta} d t \\
\leq \int_{t_{1}}^{t_{2}} \frac{M_{0}}{\eta} d t=\frac{M_{0}}{\eta}\left(t_{2}-t_{1}\right),
\end{gathered}
$$

where $M_{0}=\operatorname{Max}_{u \in N}\left|G_{5}(u)\right|$. Then

$$
\begin{aligned}
S\left(u_{1}\right)-S\left(u_{0}\right) & \geq \frac{1}{8 \alpha} b M^{-1} \varepsilon^{2}\left(t_{2}-t_{1}\right) \geq \frac{1}{8} b \varepsilon^{2} m_{0} M^{-1} M_{0}^{-1} \frac{\eta}{\alpha} \\
& =\frac{1}{80} m_{0} \varepsilon^{2} a^{2} M^{-3} M_{0}^{-1} \frac{\eta}{\sigma^{-1}}
\end{aligned}
$$

which is impossible if $\frac{\eta}{\sigma-1}$ is large. Thus such an $m_{1}>1$ exists too. The proof for the case $u_{2}$ and $u_{3}$ can be done similarly.

As we mentioned in $\S 3$, we assume that $\chi \rightarrow 0$ as $\alpha \rightarrow 0$. Moreover, $\chi \alpha^{-1}$ remains bounded from the above during the limiting process. In the following theorem we use these assumptions.

Theorem 6.1. Suppose in the system (2.1), $\eta, k, \sigma^{-1}>0$ are fixed and $0<$ $\alpha \leq \alpha_{0}, 0<\chi \leq \chi_{0},(\alpha, \chi) \rightarrow 0$ such that $0<\chi \alpha^{-1} \leq m$ for some constant $m$. For fixed $\alpha, \chi$, let $\gamma_{\alpha}(t)$ be a complete orbit of the system (2.1) which is running from $u_{0}$ to $u_{1}$. If $\sigma^{-1} \eta^{-1}<\min \left(m_{1}^{-1}, \frac{1}{2} m_{0} m_{2}^{-1}\right)$, where $m_{2}=\operatorname{Max}_{u \in N_{01}} V^{-1}\left|G_{5}(u)\right|$, and $m_{0}$ and $m_{1}$ are given in Lemma 6.2, then $\varlimsup\left\{\gamma_{\alpha}(t): t \in R\right\} \backslash\left\{u_{0}, u_{1}\right\}$ is the unique heteroclinic orbit of the system (3.5) running from $u_{0}$ to $u_{1}$ corresponding to the viscosity parameters $\eta, k$ and $\sigma^{-1}$.

Proof. We will show that the system (2.1) (as (6.1)) together with $u_{0}$ and $u_{1}$ (as the rest points), the isolating neighbourhood $N_{01}$ (as $\left.\Omega\right),(\alpha, \chi)$ (as $\mu$ ), 


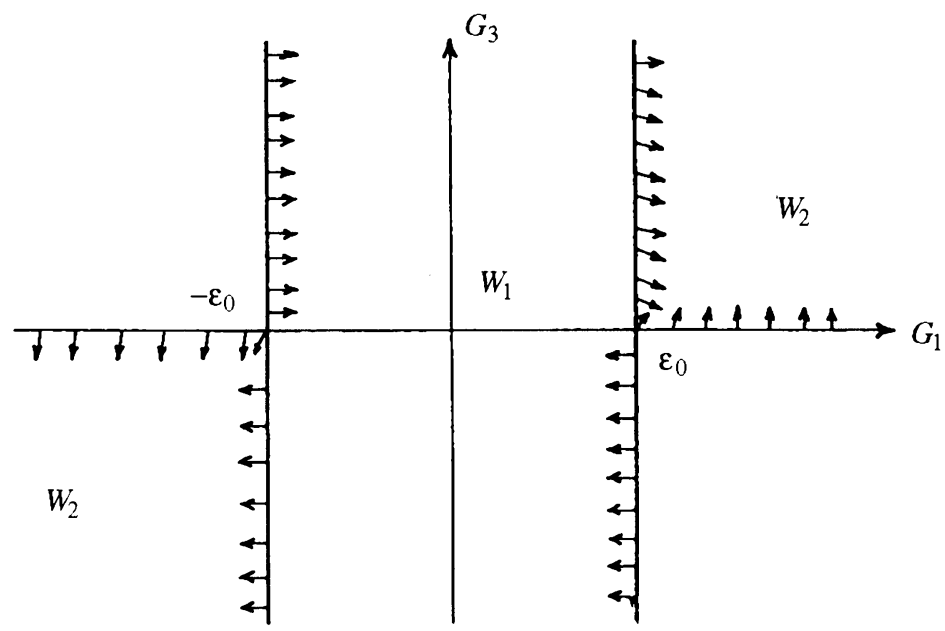

FIGURE 6.1. The arrows show the direction of the flow on the boundaries of $W_{1}$ and $W_{2}$

$\left(G_{5}(u), G_{6}(u)\right)^{\mathrm{T}}($ as $f), V^{-1}\left(G_{1}(u), G_{2}(u), G_{3}(u), G_{4}(u)\right)^{\mathrm{T}}($ as $g)$ and the system (3.5) (as (6.2)) satisfy conditions $C_{1}-C_{7}$ of Lemma 6.1.

Conditions $C_{1}, C_{2}$ and $C_{3}$ obviously hold. In order to see that condition $C_{4}$ holds, for given $\varepsilon_{0}>0$, we define

$$
W_{3}=\left\{u \in N_{01}:\left|G_{3}(u)\right|<\varepsilon_{0}\right\} .
$$

If we differentiate $G_{3}(u)$ along the orbits of the system $(2.1)$ we get

$$
\frac{d G_{3}}{d t}=\frac{1}{\alpha V}\left[\left(V-\delta^{2}+\frac{\sigma^{-1}}{\eta} \frac{G_{5}}{V}\right) G_{1}-\frac{\sigma^{-1}}{\alpha} \frac{G_{3}}{V}+\frac{\alpha}{\eta} V B_{2} G_{5}-\frac{\chi}{\alpha} \delta G_{4}\right] .
$$

Since $0<\frac{\chi}{\alpha} \leq m$ and $\sigma^{-1} \eta^{-1} \leq m_{1}^{-1}$, the flow gets into $W_{3}$ on $\partial W_{3}$ for small values of $\alpha$ and $\chi$. Since $u_{0}$ and $u_{1}$ are in $W_{3}, G_{3}\left(\gamma_{\alpha}(t)\right) \rightarrow 0$ uniformly on $R$ as $(\alpha, \chi) \rightarrow 0$. Similarly $G_{4}\left(\gamma_{\alpha}(t)\right) \rightarrow 0$ uniformly as $(\alpha, \chi) \rightarrow 0$. In order to see that $G_{1}\left(\gamma_{\alpha}(t)\right) \rightarrow 0$ uniformly, for small values of $\alpha$ and given $\varepsilon_{0}>0$ we define

$$
\begin{aligned}
& W_{1}=\left\{u \in N_{01}: V-\delta^{2} \geq m_{0},\left|G_{1}(u)\right|<\varepsilon_{0}\right\}, \\
& W_{2}=\left\{u \in N_{01}: V-\delta^{2} \geq m_{0},\left|G_{1}(u)\right|>\varepsilon_{0}, G_{1}(u) G_{3}(u)>0\right\} .
\end{aligned}
$$

Now $G_{4}\left(\gamma_{\alpha}(t)\right) \rightarrow 0,0<\frac{\chi}{\alpha} \leq m$ and by Lemma 6.2, along $\gamma_{\alpha}(t), V-\delta^{2}+$ $\frac{\sigma^{-1}}{\eta} \frac{G_{5}}{V} \geq \frac{m_{0}}{2}$. Thus $\frac{d G_{1}}{d t}=\frac{G_{3}}{\alpha V}$ and (6.4) imply that the flow gets into $W_{2}$ on $\partial W_{2}$, goes out of $W_{1}$ on $\partial W_{1} \cap \partial W_{2}$ and gets into $W_{1}$ on $\partial W_{1} \backslash \partial W_{2}$ for small values of $\alpha$ and $\chi$. These results are illustrated in the $\left(G_{1}, G_{3}\right)$ plane in Figure 6.1, where the arrows show the direction of the flow on $\partial W_{1}$ and $\partial W_{2}$. We claim that for small values of $\alpha$ and $\chi, \gamma_{\alpha}(t)$ must lie in $W_{1}$. To see this, note that, if this orbit leaves $W_{1}$, then it must spend a time interval inside of $W_{2}$. However, this orbit cannot leave $W_{2}$. Thus this orbit cannot connect $u_{0}$ to $u_{1}$ which are in $W_{1}$. Hence $\left|G_{1}\left(\gamma_{\alpha}(t)\right)\right|<\varepsilon_{0}$ for small values of $\alpha$ and $\chi$. Thus $G_{1}\left(\gamma_{\alpha}(t)\right) \rightarrow 0$ uniformly in $R$ as $(\alpha, \chi) \rightarrow 0$. Similarly $G_{2}\left(\gamma_{\alpha}(t)\right) \rightarrow 0$ uniformly in $R$ as $(\alpha, \chi) \rightarrow 0$. Therefore $\left[V_{\alpha}(t)\right]^{-1} G_{i}\left(\gamma_{\alpha}(t)\right) \rightarrow 0$ uniformly as $(\alpha, \chi) \rightarrow 0,1 \leq i \leq 4$. Hence condition $C_{4}$ holds. 
Now we will show that condition $C_{5}$ holds. To do this, for simplicity, we set $G_{i}\left(\gamma_{\alpha}(t)\right)=z_{i \alpha}(t), 1 \leq i \leq 4$. Then for $i=1,2$ we have

$$
\left|y_{i \alpha}(t)-y_{i \alpha^{\prime}}(t)\right| \leq 2\left|z_{i \alpha}(t)-z_{i \alpha^{\prime}}(t)\right| .
$$

Also from the third equation of (2.2) we can write

$$
\sigma^{-1} \dot{B}_{2 \alpha}-\left(V-\delta^{2}\right) B_{2 \alpha}=\varepsilon-z_{3 \alpha}(t)-\chi \alpha^{-1} \delta y_{3 \alpha}(t) .
$$

Then from this we obtain

$$
h_{\alpha}(t) B_{2 \alpha}(t)=B_{2 \alpha}(0)+\sigma \int_{0}^{t}\left[\varepsilon-z_{3 \alpha}(s)-\chi \alpha^{-1} \delta y_{3 \alpha}(s)\right] h_{\alpha}(s) d s
$$

where $h_{\alpha}(s)=e^{-\sigma} \int_{0}^{t}\left[V_{\alpha}(s)-\delta^{2}\right] d s$. Thus

$$
\begin{aligned}
h_{\alpha}(t) B_{2 \alpha}(t)-h_{\alpha^{\prime}}(t) B_{2 \alpha^{\prime}}(t)= & B_{2 \alpha}(0)-B_{2 \alpha^{\prime}}(0)+\varepsilon \sigma \int_{0}^{t}\left[h_{\alpha}(s)-h_{\alpha^{\prime}}(s)\right] d s \\
& -\sigma \int_{0}^{t}\left[z_{3 \alpha}(s) h_{\alpha}(s)-z_{3 \alpha^{\prime}}(s) h_{\alpha^{\prime}}(s)\right] d s \\
& -\sigma \chi \alpha^{-1} \delta \int_{0}^{t}\left[y_{3 \alpha}(s) h_{\alpha}(s)-y_{3 \alpha^{\prime}}(s) h_{\alpha^{\prime}}(s)\right] d s .
\end{aligned}
$$

Thus for given $a>0$, there exist constants $b_{i}, 1 \leq i \leq 4$, such that for $t \in[0, a]$,

$$
\begin{aligned}
h_{\alpha}(t)\left|B_{2 \alpha}(t)-B_{2 \alpha^{\prime}}(t)\right| \leq\left|B_{2 \alpha^{\prime}}(t)\right|\left|h_{\alpha}(t)-h_{\alpha^{\prime}}(t)\right|+\left|B_{2 \alpha}(0)-B_{2 \alpha^{\prime}}(0)\right| \\
\quad+\varepsilon \sigma \int_{0}^{t}\left|h_{\alpha}(s)-h_{\alpha^{\prime}}(s)\right| d s+\sigma \int_{0}^{t}\left|z_{3 \alpha}(s) h_{\alpha}(s)-z_{3 \alpha^{\prime}}(s) h_{\alpha^{\prime}}(s)\right| d s \\
\quad+\sigma \chi \alpha^{-1} \delta \int_{0}^{t}\left|y_{3 \alpha}(s) h_{\alpha}(s)-y_{3 \alpha^{\prime}} h_{\alpha^{\prime}}(s)\right| d s \\
\leq\left|B_{2 \alpha}(0)-B_{2 \alpha^{\prime}}(0)\right|+b_{1} \int_{0}^{t}\left|V_{\alpha}(s)-V_{\alpha^{\prime}}(s)\right| d s \\
\quad+b_{2} \int_{0}^{t} \int_{0}^{s}\left|V_{\alpha}(r)-V_{\alpha^{\prime}}(r)\right| d r d s+b_{3} \int_{0}^{t}\left|z_{3 \alpha}(s)-z_{3 \alpha^{\prime}}(s)\right| d s \\
\quad+b_{4} \int_{0}^{t}\left|y_{3 \alpha}(s)-y_{3 \alpha^{\prime}}(s)\right| d s .
\end{aligned}
$$

Obviously similar inequalities hold for $t \in[-a, 0]$. By using a similar argument we can show that the same inequality is satisfied for $\left|B_{3 \alpha}(t)-B_{3 \alpha^{\prime}}(t)\right|$. Thus condition $C_{5}$ holds.

Now suppose $\left(\alpha_{k}, \chi_{k}\right) \rightarrow 0$ and

$$
\begin{aligned}
\gamma_{\alpha_{k}}(t) & =\left(B_{2 k}(t), B_{3 k}(t), y_{2 k}(t), y_{3 k}(t), V_{k}(t), T_{k}(t)\right)^{\mathrm{T}} \rightarrow \gamma(t) \\
& =\left(B_{2}(t), B_{3}(t), y_{2}(t), y_{3}(t), V(t), T(t)\right)^{\mathrm{T}},
\end{aligned}
$$

uniformly in $[-a, a]$. The first four equalities of (2.2) for $\gamma_{\alpha_{k}}(t)$ are

$$
\begin{gathered}
y_{2 k}(t)=G_{1}\left(\gamma_{\alpha_{k}}(t)\right), \\
y_{3 k}(t)=G_{2}\left(\gamma_{\alpha_{k}}(t)\right), \\
\varepsilon+\left[V_{k}(t)-\delta^{2}\right] B_{2 k}(t)-\chi \alpha^{-1} \delta y_{3 k}(t)-\sigma^{-1} \dot{B}_{2 k}(t)=G_{3}\left(\gamma_{\alpha_{k}}(t)\right), \\
{\left[V_{k}(t)-\delta^{2}\right] B_{3 k}(t)+\chi \alpha^{-1} \delta y_{2 k}(t)-\sigma^{-1} \dot{B}_{3 k}(t)=G_{4}\left(\gamma_{\alpha_{k}}(t)\right) .}
\end{gathered}
$$


Now $G_{i}\left(\gamma_{\alpha_{k}}(t)\right) \rightarrow 0$ and $\gamma_{\alpha_{k}}(t) \rightarrow \gamma(t)$ uniformly on $[-a, a]$, hence $y_{i k}(t) \rightarrow$ $0, i=2,3$. Using these and $0<\chi \alpha^{-1} \leq m$ in the last two equalities of (6.5) we obtain $\left\{\dot{B}_{i k}(t)\right\}$ is uniformly convergent on $[-a, a], i=2,3$, and

$$
\begin{aligned}
\varepsilon+\left[V(t)-\delta^{2}\right] B_{2}(t)-\sigma^{-1} \dot{B}_{2}(t) & =0, \\
{\left[V(t)-\delta^{2}\right] B_{3}(t)-\sigma^{-1} \dot{B}_{3}(t) } & =0 .
\end{aligned}
$$

Thus, we obtain

$$
\bar{g}\left(u, \dot{B}_{2}, \dot{B}_{3}\right)=\left(0,0, \varepsilon+\left(V-\delta^{2}\right) B_{2}-\sigma^{-1} \dot{B}_{2},\left(V-\delta^{2}\right) B_{3}-\sigma^{-1} \dot{B}_{3}\right)^{\mathrm{T}},
$$

and the corresponding system of equations to the system (6.2) is (3.5). Since the system (3.5) is gradient-like with respect to $P(u)$ and $P\left(u_{0}\right)<P\left(u_{1}\right)$, condition $C_{7}$ holds too. Thus by Lemma $6.1, \overline{\lim }\left\{\gamma_{\alpha}(t)\right\} \backslash\left\{u_{0}, u_{1}\right\}$ is a set of union of the heteroclinic orbits between $u_{0}$ and $u_{1}$. On the other hand the stable manifold at $u_{1}$ for the system (3.5) has dimension one; thus this system admits a unique orbit between $u_{0}$ and $u_{1}$. This completes the proof.

Corollary 6.1. Theorem 6.1 is valid for the case $\sigma^{-1}=0$, if $\chi \alpha^{-1} \rightarrow 0$ as $\alpha \rightarrow 0$.

Proof. It suffices to prove that condition $C_{4}$ of Lemma 6.1 holds. First of all note that in Case 2 of the proof of Lemma 3.4, we showed that for small values of $\alpha$ and $\chi=0$, the heteroclinic orbits of the system (2.1) between $u_{0}$ and $u_{1}$ lie in a compact set in the region $V>\delta^{2}$. This conclusion is valid for small values of $\alpha$ whenever $\chi$ is small too, as $\chi$ is a regular parameter. Thus we may assume that along these orbits $V-\delta^{2} \geq m_{0}$ for some constant $m_{0}$ whenever $\alpha$ and $\chi$ are small. Now differentiating $G_{1}(u), G_{3}(u)$ and $\left|G_{3}(u)\right|+\frac{1}{2} m_{0}\left|G_{1}(u)\right|$ along the orbits of the system (2.1) yield

$$
\begin{gathered}
\frac{d G_{1}}{d t}=\frac{G_{3}}{\alpha V}, \quad \frac{d G_{3}}{d t}=\frac{1}{\alpha V}\left[\left(V-\delta^{2}\right) G_{1}+\frac{\alpha}{\eta} B_{2} V G_{5}-\frac{\chi}{\alpha} \delta G_{4}\right] \\
\frac{d}{d t}\left( \pm G_{3} \pm \frac{1}{2} m_{0} G_{1}\right)= \pm \frac{1}{\alpha V}\left[\left(V-\delta^{2}\right) G_{1} \pm \frac{1}{2} m_{0} G_{3}+\frac{\alpha}{\eta} B_{2} V G_{5}-\frac{\chi}{\alpha} \delta G_{4}\right] .
\end{gathered}
$$

Since $V-\delta^{2} \geq m_{0}$ and $\underset{\alpha}{\chi} \rightarrow 0$, the flow gets into $W_{2}$ on $\partial W_{2}$, goes out of $W_{1}$ on $\partial W_{1} \cap \partial W_{2}$ and gets into $W_{1}$ on $\partial W_{1} \backslash \partial W_{2}$ for small values of $\alpha$ and $\chi$, where

$$
\begin{aligned}
& W_{1}=\left\{u \in N_{01}: V-\delta^{2} \geq \frac{1}{2} m_{0},\left|G_{3}(u)\right|+\frac{1}{2} m_{0}\left|G_{1}(u)\right|<\varepsilon_{0}\right\}, \\
& W_{2}=\left\{u \in N_{01}: V-\delta^{2} \geq \frac{1}{2} m_{0},\right. \\
& \left.\qquad G_{3}(u)\left|+\frac{1}{2} m_{0}\right| G_{1}(u) \mid>\varepsilon_{0}, G_{1}(u) \cdot G_{3}(u)>0\right\} .
\end{aligned}
$$

These results are illustrated in Figure 6.2. Thus $\gamma_{\alpha}(t)$ must lie in $W_{1}$ for small values of $\alpha$ and $\chi$. Similar to the proof of Theorem 6.1, it follows that condition $C_{4}$ holds.

Let $\lambda=\left(\alpha, \eta, k, \sigma^{-1}, \chi\right)>0$ and $\gamma_{\lambda}(t)$ be a connecting orbit between $u_{i}$ and $u_{i+1}, i=0,2$, corresponding to $\lambda$. Suppose $\lambda \rightarrow \lambda_{0}=\left(\alpha_{0}, \eta_{0}, k_{0}, \sigma_{0}^{-1}\right.$, 


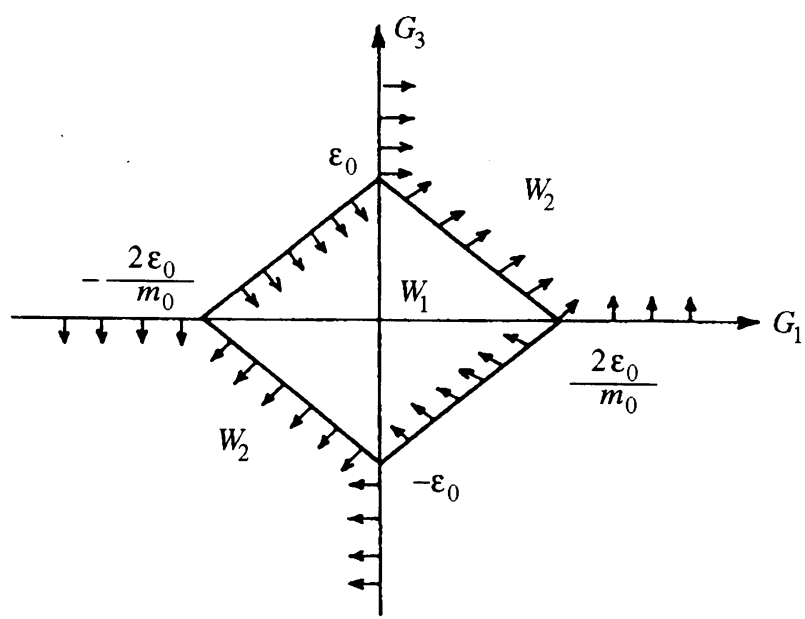

Figure 6.2. The arrows show the direction of the flow on the boundaries of $W_{1}$ and $W_{2}$

$\left.\chi_{0}\right)$ where some of the components of $\lambda_{0}$ are zero and the others are the same as $\lambda$. Then the set $S_{i}=\lim \sup _{\lambda_{0}<\lambda<\lambda_{1}}\left\{\gamma_{\lambda}(t): t \in R\right\} \backslash\left\{u_{i}, u_{i+1}\right\}, i=0,2$, is a nonempty union of complete orbits of the system (2.1) corresponding to $\lambda_{0}$, running from $u_{i}$ to $u_{i+1}, i=0,2$. Here $\lambda_{1}$ is an arbitrary upper bound for the $\lambda, s$. In order to prove this, it suffices to show this claim is valid for each sequence $\lambda_{m}$ as $\lambda_{m} \rightarrow \lambda_{0}$.

First of all suppose $\lambda_{m}=\left(\alpha, \eta, k, \sigma_{m}^{-1}, \chi_{m}\right), \lambda_{0}=(\alpha, \eta, k, 0,0)$ and $\lambda_{m} \rightarrow \lambda_{0}$. Then the system (2.1) corresponding to $\lambda_{m}$ and $\lambda_{0}$ can be written

$$
\begin{aligned}
& \dot{u}=A^{-1}\left(G_{1}(u), G_{2}(u), G_{3}^{m}(u), G_{4}^{m}(u), G_{5}(u), G_{6}(u)\right)^{\mathrm{T}}, \\
& \dot{u}=A^{-1}\left(G_{1}(u), G_{2}(u), G_{3}^{0}(u), G_{4}^{0}(u), G_{5}(u), G_{6}(u)\right)^{\mathrm{T}}
\end{aligned}
$$

respectively, where

$$
\begin{aligned}
G_{3}^{m} & =\varepsilon+\left(V-\delta^{2}\right) B_{2}-\frac{\chi_{m}}{\alpha} \delta y_{3}-\frac{\sigma_{m}^{-1}}{\alpha V} y_{2}, \\
G_{4}^{m} & =\left(V-\delta^{2}\right) B_{3}+\frac{\chi_{m}}{\alpha} \delta y_{2}-\frac{\sigma_{m}^{-1}}{\alpha V} y_{3}, \\
G_{3}^{0} & =\varepsilon+\left(V-\delta^{2}\right) B_{2}, \quad G_{4}^{0}=\left(V-\delta^{2}\right) B_{3} .
\end{aligned}
$$

Thus

$$
\begin{gathered}
\left|A^{-1}\left(G_{1}, G_{2}, G_{3}^{m}, G_{4}^{m}, G_{5}, G_{6}\right)^{T}-A^{-1}\left(G_{1}, G_{2}, G_{3}^{0}, G_{4}^{0}, G_{5}, G_{6}\right)^{T}\right| \\
\quad \leq \frac{1}{\alpha^{2} V^{2}}\left[\left(\chi_{m}+\sigma_{m}^{-1}\right)\left(\left|y_{2}\right|+\left|y_{3}\right|\right)\right] \leq \frac{2 M}{\alpha^{2} a^{2}}\left(\chi_{m}+\sigma_{m}^{-1}\right) \rightarrow 0
\end{gathered}
$$

where $M$ and $a$ are as in Theorem 3.3. Thus all of the conditions of Lemma 3.1.2 in [13] are fulfilled. Hence by that lemma $S_{i}$ is a nonempty union of heteroclinic orbits between $u_{i}$ and $u_{i+1}, i=0,2$, corresponding to $\lambda_{0}$. The above argument shows that if $\lambda_{m}=\left(\alpha, \eta, k, \sigma_{m}^{-1}, \chi\right)$ and $\lambda_{0}=(\alpha, \eta, k, 0, \chi)$ or $\lambda_{m}=\left(\alpha, \eta, k, \sigma^{-1}, \chi_{m}\right)$ and $\lambda_{0}=\left(\alpha, \eta, k, \sigma^{-1}, 0\right)$, then the similar conclusions are valid. 
Now suppose $\lambda=\left(\alpha, \eta, k, \sigma^{-1}, \chi\right), \lambda_{0}=\left(\alpha, \eta, 0, \sigma^{-1}, \chi\right), \lambda \rightarrow \lambda_{0}$. We will show that, in this case, conditions $C_{1}-C_{7}$ of Lemma 6.1 are satisfied. We need only to check condition $C_{4}$. To do this, for given $\varepsilon_{0}>0$, we define

$$
W=\left\{u \in N:\left|G_{6}(u)\right|<\varepsilon_{0}\right\} .
$$

If we differentiate $G_{6}(u)$ along the orbits and using $e_{V}=T S_{V}-p, e_{T}=T S_{T}$ we obtain

$$
\frac{d G_{6}}{d t}=T S_{T} \frac{G_{6}}{k}+T S_{V} \frac{G_{5}}{\eta}-\frac{G_{5}^{2}}{\eta}-\frac{\sigma^{-1}}{\alpha^{2} V^{2}}\left(G_{1}^{2}+G_{2}^{2}\right) .
$$

Since $\min _{u \in N} T S_{T}>0$, it follows that the flow goes out of $W$ on $\partial W$ for small values of $k$. Thus condition $C_{4}$ holds. Hence by Lemma $6.1, S_{i}$ is a nonempty union of heteroclinic orbits.

Finally, suppose $\lambda \rightarrow \lambda_{0}=\left(\alpha, 0, k, \sigma^{-1}, \chi\right)$. If $1+p_{V}(u)>0$ in $N$ (which is possible when $\left|u_{i}-u_{i+1}\right|$ is small $\left.i=0,2\right)$, similar to the above, we can show that conditions $C_{1}-C_{7}$ of Lemma 6.1 hold.

Therefore we have proved the following theorem.

Theorem 6.2. Let $\lambda=\left(\alpha, \eta, k, \sigma^{-1}, \chi\right)>0$ and $\lambda_{0}=\left(\alpha, \eta_{0}, k_{0}, \sigma_{0}^{-1}, \chi_{0}\right)$ where one of the last four components of $\lambda_{0}$ is zero and the others are the same as $\lambda$. Suppose $\gamma_{\lambda}(t)$ is a heteroclinic orbit of the system (2.1) corresponding to $\lambda$, between $u_{i}$ and $u_{i+1}, i=0,2 ; \lambda \rightarrow \lambda_{0}$; and for the case $\eta_{0}=0,\left|u_{i}-u_{i+1}\right|$ is small. Then

$$
S_{i}=\limsup _{\lambda_{0}<\lambda<\lambda_{1}}\left\{\gamma_{\lambda}(t): t \in R\right\} \backslash\left\{u_{i}, u_{i+1}\right\}, \quad i=0,2,
$$

is a nonempty union of the heteroclinic orbits of the system (2.1) corresponding to $\lambda_{0}$. Here $\lambda_{1}$ is an upper bound for $\lambda, s$.

\section{ACKNOWLEDGMENTS}

The author would like to thank Professor Abdus Salam, the International Atomic Energy Agency, UNESCO and the International Centre for Theoretical Physics, Trieste, for supporting this research.

\section{REFERENCES}

1. J. E. Anderson, Magnetohydrodynamics shock waves, M.I.T. Press, Cambridge, Mass., 1963.

2. C. C. Conley, Isolated invariant sets and Morse index, C.B.M.S. Regional Conf. Ser. Math., no. 38, Amer. Math. Soc., Providence, R.I., 1978.

3. C. C. Conley and R. Easton, Isolated invariant sets and isolating blocks, Trans. Amer. Math. Soc. 158 (1971), 35-61.

4. C. C. Conley and J. A. Smoller, On the structure of magnetohydrodynamic shock waves, Comm. Pure Appl. Math. 27 (1974), 367-375.

5. _ On the structure of magnetohydrodynamic shock waves. II, J. Math. Pures Appl. 54 (1975), 429-444.

6. _ Viscosity matrices for two-dimensional nonlinear hyperbolic system, Comm. Pure Appl. Math. 23 (1970), 867-884.

7. _ Shock waves as limits of progressive wave solutions of higher order equations, Comm. Pure Appl. Math. 24 (1971), 459-472.

8. Topological methods in the theory of shock waves, Berkeley Sympos. on Partial Differential Equations Summer, 1971. 
9. P. Germain, A model of some plasma shock structures, Magneto-Fluid and Plasma Dynamics, Proc. Sympos. Appl. Math., vol. 18, Amer. Math. Soc., Providence, RI, 1967, pp. 17-45.

10. __ Shock waves, jump relations, and structure, Adv. in Appl. Mech. 12 (1972), 131-194.

11. __ Shock waves and shock waves structure in magneto-fluid dynamics, Rev. Modern Phys. 32 (1960), 951-958.

12. M. Hesaaraki, The structure of MFD shock waves in a model of two fluids, Nonlinearity 6 (1993), 1-24.

13. _ The structure of shock waves in magnetohydrodynamics, Mem. Amer. Math. Soc., vol. 49, no. 302, 1984.

14. _ The structure of shock waves in magnetohydrodynamics for purely transverse magnetic fields, SIAM J. Appl. Math. 51 (1991), 412-428.

15. (1993), 377-404.

16. A. G. Kulikovskiy and G. A. Lyubimov, Magnetohydrodynamics, Addison-Wesley, Reading, MaA, 1965.

17. R. Peyret, Sur la structure due choc lent dans un schéma à deus fluids non dissipatif, C. R. Acad. Sci. Paris Sér. I. Math. 258 (1964), 2973-2976.

18. _ Sur la structure du choc lent dans un schéma à deux fluides avec dissipation, C. R. Acad. Sci. Paris Ser. I Math. 258 (1964), 3178-3181.

19. D. Salamon, Connected simple systems and the Conley index of isolated invariant sets, Trans. Amer. Math. Soc. 291 (1985), 1-41.

20. J. A. Smoller, Shock waves and reaction diffusion equations, Springer-Verlag, New York, Heidelberg, and Berlin, 1983.

21. J. A. Smoller and C. C. Conley, Viscosity matrices for two-dimensional nonlinear hyperbolic system. II, Amer. J. Math. 44 (1972), 631-650.

22. __ Shock waves as limits of progressive wave solutions of higher order equations. II, Comm. Pure Appl. Math. 25 (1975), 133-146.

23. P. Thompson, Compressible-fluid dynamics, McGraw-Hill, New York, 1972.

24. G. T. Whyburn, Analytic topology, Amer. Math. Soc. Colloq. Publ., vol. 28, Amer. Math. Soc., Providence, R.I., 1942.

International Centre for Theoretical Physics, Trieste, Italy

Current address: Department of Mathematical Sciences, Sharif University of Technology, Tehran, Iran 